\title{
Projecting the impact of anal intercourse on HIV transmission among heterosexuals in high HIV prevalence settings.
}

\author{
Noble. J. Malunguza ${ }^{1 *}$, Senelani D. Hove-Musekwa ${ }^{1}$, Zindoga Mukandavire ${ }^{2}$, \\ ${ }^{1}$ Department of Applied Mathematics, National University of Science and Technology, Bulawayo, Zimbabwe \\ ${ }^{2}$ Social and Mathematical Epidemiology Group, London School of Hygiene and Tropical Medicine, London, UK
}

\begin{abstract}
Whereas penile vaginal intercourse (VI) is thought to be the dominant mode of HIV transmission in sub-Saharan Africa, cross-sectional studies in the region indicate the preponderance of heterosexual anal intercourse (AI) among high activity groups. A dynamic, heterosexual core group model with risk of infection through both vaginal and anal pathways is formulated and comprehensively analysed. The model is coupled to a general population model and fitted to HIV prevalence data for Zimbabwe in order to explore the parameter space related to heterosexual AI. The model fit supports a core group size ranging between $5-20 \%$ and exposure risk to AI in excess of $50 \%$. The control effort signified by the reproductive number $\left(\mathcal{R}_{A}\right)$ at commencement of the epidemic corresponds to $\mathcal{R}_{0}=4.40$. With the contribution of heterosexual AI to Zimbabwe's (and that of the sub-Saharan African region) epidemic unknown, the study apportioned infections between the two infection pathways. Incidence due to VI ranged from $2-4.5 \%$ and $0.5-2.7 \%$ from heterosexual AI. The study estimates infection probabilities of between $15-35 \%$ for both receptive and infective AI. By quantifying the incidence due to VI and AI risks, we put emphasis on the necessity for targeted interventions. To project the potential impact of heterosexual AI in high HIV prevalence settings, we raised the core group size to $20 \%$ in the year 2010 coinciding with reported heterosexual AI prevalence outside of Africa and allowed the proportion of infection risk associated with AI to vary. Prevalence and incidence projections were made up to the year 2020 starting from a baseline value of zero exposure to AI transmission risk, progressively increasing exposure to $50 \%$ and $70 \%$ respectively. A $50 \%$ exposure to AI would result in HIV prevalence scaling up by $23 \%$ from the baseline values in year 2020 . Increasing exposure to $70 \%$ was projected to increase HIV prevalence by $38 \%$ in year 2020. The HIV infection risks associated with AI are recognised and inform HIV policing for men who have sex with men, yet the same risks are ignored in HIV policing for heterosexuals. This study highlights the potential danger of increasing prevalence of heterosexual AI in settings with high HIV prevalence. Evolving and globally cross pollinating sexual behaviors compel for dovetailing HIV policy making with sexology.
\end{abstract}

Key words: HIV model, transmission, vaginal intercourse, heterosexual anal intercourse.

\section{Introduction}

Heterosexuality and by extrapolation, vaginal-penile sexual intercourse, is thought to account for the bulk of HIV transmissions in Africa [27, 40,65]. Although AI is known to confer higher odds for infection $[16,37,40,68]$, earlier studies $[29,53]$ estimated prevalence of AI in the general population to be very small $(<1 \%)$. Recent studies have however noted its significant practise amongst high activity groups such as prostitutes, patrons of informal alcohol serving establishments (referred to as shebeens) and students $[1,4,8,24,39,40,54]$.

\footnotetext{
${ }^{*}$ Correspondence: Noble. J. Malunguza, Email: nmalunguza@gmail.com,nmalunguza@nust.ac.zw
} 
In the developed world, AI in the general population is well documented $[22,43,47,49,80]$. Results in [47] suggest that $25 \%$ of heterosexuals in the US has engaged in AI and for 1 in 12 , it is an episodic practice. A study of young adults in the US aged 18-26 years found that $23 \%$ had engaged in AI, and $22 \%$ reported AI with their current partner in the past three months [43]. In another study in California [22], $8 \%$ of males and $6 \%$ of females reported having had unprotected AI at least once a month. Amongst North American college women, survey results showed an $18.6 \%$ prevalence of AI, [49] while studies in [80] estimated that 25\% of American women and their male partners engaged in AI episodically, with $10 \%$ engaged in it repeatedly. In sub-Saharan Africa, AI is highly stigmatized and possibly underreported $[44,66]$ and therefore difficult to quantify. It is thus even more difficult to assess its subsequent contribution to HIV transmission within the heterosexual population. In the region AI is reportedly practiced by heterosexual couples to prevent pregnancy, beliefs that AI will prevent HIV transmission, relationship security, hiding infidelity, preserve a woman's virginity, and obtain pleasure as well as being an alternative to VI during menstruation [21].

The practice of AI appears to be ubiquitous across all heterosexual populations, age groups and countries, with studies reporting in excess of $20 \%$ of selected populations in Africa and elsewhere having engaged in AI [8]. Among female sex workers in sub-Saharan Africa, prevalence ranged from $0-43 \%[1,4]$ reporting having practiced AI and $40 \%$ of female sex workers from Kenya reporting AI within the previous 3 months. In comparison, among the general population in sub-Saharan Africa, prevalence ranged from $2-20 \%$ reporting ever having had AI. In populations with high HIV incidences in sub-Saharan Africa [74], AI prevalence rates amongst young adults ranged from $1.3 \%$ of South African high school students reporting ever having had AI [24] to $56 \%$ and $64 \%$ of university students in Nigeria and Zimbabwe, respectively, reporting its practice in the previous 2 months $[3,8]$. In a survey of shebeen patrons (people who drink in shebeens) in South Africa [39], $15 \%$ of men and 11\% of women reported AI in the previous month, with $8 \%$ of men and $7 \%$ of women practising unprotected AI. In another study Kalichman et al. [40] report overall prevalence of AI of $14 \%$ amongst men and $10 \%$ amongst women in the previous 3 months with $56 \%$ of respondents having done it on at least $50 \%$ of all sex occasions. A study of Kenyan men who had sold sex in the last 30 days [54], revealed that $45 \%$ had done so with a female client and $86 \%$ with a non-paying female partner. In the study, $66 \%$ and $43 \%$ of male sex workers had AI with a female client and non-paying partner respectively and thus indicating a general link between risky repetitive AI practitioners and the low risk general population whose practice of AI might be episodic. Table 1 gives a summary of the prevalence of AI in the some of the sub-populations in the region. However, these studies do not give HIV prevalence in the sub-populatitons viz a viz prevalence in the general population.

\begin{tabular}{|l|c|l|}
\hline Prevalence of AI & Population & Source \\
\hline $0-43 \%$ & CSW & {$[1,4]$} \\
\hline $13-56 \%$ & Students & {$[24]$} \\
\hline $11-15 \%$ & Shebeen Patrons & {$[39]$} \\
\hline $10-14 \%$ & Convenience samples & {$[40]$} \\
\hline
\end{tabular}

Table 1: Prevalence of AI in various sub-populations in the region.

The association between heterosexual AI and HIV infection is not well studied, despite the understanding that AI presents a considerably higher risk of HIV transmission than VI [53]. Results of a study, [38], showed that students who were infected with HIV were 3 times more likely to have engaged in AI than those students who were not infected. Significant heterogeneity between infectiousness estimates due to AI were observed in [7] with suggestions that a greater understanding of the role played by AI in heterosexual settings may be vital for HIV prevention. In [5] people reporting engaging in AI used condoms less frequently during vaginal intercourse in the previous 3 months than did other people: $65.3 \%$ 
of people engaging in AI used condoms $25 \%$ of the time or less, compared with $45.2 \%$ of the people who did not engage in AI. It was reported in [47] that the risk of having unprotected AI varied greatly by risk group with underlying power dynamics greatly influencing female sexual decision-making. A strong correlation between unprotected AI and sexual violence was noted in [8]. Intimate partner violence was directly correlated to AI in young adult relationships [45] and physical violence occurred in $29 \%$ of the relationships, sexual violence in $11 \%$ and $\mathrm{AI}$ in $14 \%$. Women in physically violent relationships were at an increased risk for STDs because of their elevated exposure to unprotected AI.

The low prevalence of AI in the general population may however be offset by its greater efficiency in transmitting HIV $[16,37,40,68]$. HIV prevention strategies targeting men who have sex with men (MSM) are premised on this hightened risk which is ignored in heterosexual populations with prevention programmes limited to the penile-vaginal pathway. According to [18], the increased likelihood of mucosal disruption and trauma in the epithelium lining the rectum against the vaginal lining's epithelium, and the possible increased risk of trauma due to the muscular anal sphincter present possible reasons for the risk differential. A study by [48] estimated the probability of HIV infection per act of receptive AI at $3.4 \%$ versus less than $0.01 \%$ per act of vaginal intercourse and also found a 5.1 increased odds of HIV infection from anal compared with vaginal intercourse [23]. In [16], the risk estimates for HIV transmission, per sex act, range widely, from $0.5-3.38 \%$ (with mid-range estimates of $1.4-1.69 \%$ ) for receptive AI, $0.06-0.16 \%$ for insertive AI, $0.08-0.19 \%$ for receptive vaginal intercourse and $0.05-0.1 \%$ for insertive vaginal intercourse.

Although studies show the practice of AI as being limited to small subsections of the population with many in the general population confusing AI with other non-traditional sexual forms like vaginal rear entry style positioning, oral sex and digital penetration [62], the proportions of young people reporting having had AI are not insignificant [3,7,8,24,38-40,47]. Qualitative studies indicate that AI is likely to be added to one's repertoire once initiated $[50,53,67]$, but since AI is likely an underreported activity due to stigmatisation, prevalence may in fact be higher than the data indicates. In this study, we formulate a mathematical model that couples a core group of individuals engaging in heterosexual AI to the general population. Members of this core group are deemed to interact amongst themselves but overlap and have partners in the general population. Such core group models have been used before by $[42,72]$ to study the role of transactional sex in spreading HIV, [46] to study the effect of the HIV/AIDS epidemic on the population of truck drivers in South Africa and by Hsieh et al. [26] to model the social dynamics of the sex industry. Despite knowledge that AI escalates the risk of HIV infection and that AI among high risk sub-populations in the heterosexual populations is a routine, few studies have examined the potential for AI to influence HIV dynamics in the general population. Boily [11] developed a deterministic model of heterosexual HIV transmission during vaginal intercourse, receptive AI and insertive AI, incorporating three HIV infectiousness stages and used HIV prevalence data from South Africa to compute plausible ranges of parameter values. The study estimated that $17-40 \%$ of annual infections (PAF) may be due to receptive AI. The PAF due to AI and PI was positively associated with increases in the overall fraction of all sex acts which are AI (\%AI), whereas the latter depended on the level of mixing. In the study, to make the mixing less assortative, the $\%$ acts of AI needed to be reduced to allow for more VI between AI and non-AI to occur. The study suggested that even a small fraction of AI $(<10 \%)$ in a population may be equally pivotal to overall HIV transmission in generalised epidemics, as the primary phase of infection, particularly for women. Our study is meant to add to the body of knowledge by formulation of a deterministic HIV transmission model which is validated using HIV prevalence data for Zimbabwe. Not much is known regarding the sexual dynamics of heterosexual AI in general and the region in particular. We therefore make a host of simplifying assumptions in order to come up with a mathematically tractable model while retaining relevance of the model. We begin by formulating a core group model of HIV spread among heterosexual individuals engaging in both vaginal and AI and are thus exposed to two exclusive 
pathways of infection in any one sexual encounter. The model is then coupled to a general population only exposed to infection through the penile-vaginal pathway. The two populations are assumed to mix with fluidity and the combined model is fit to HIV data for Zimbabwe.

\section{Model structure and development}

A sexually interacting heterosexual core group is divided into compartments of susceptibles and infectives categorised into $S_{i_{a}}(t)$ and $I_{i_{a}}(t), i=(f, m)$ denoting female and male with the subscript $a$, denoting the core group at time $t$ depending on whether they came into effective contact with the virus or not. Individuals who have progressed to symptomatic AIDS are assumed to be sexually inactive and thus not distinguished and are categorised in the AIDS class which is deemed non-interacting. This study assumes removal by symptomatic progression to AIDS. Since the reported core group size is small [29,53], and nowadays clinical latency can last an average of 12 years without treatment and several decades if ART is administered, [2,64], it is convenient to assume immediate replenishment from the general population, of loses due to AIDS progression as well as loses due to any other decrement. Thus the core group size is assumed constant ( but treated as a fit variable in the parameterisation of the model in Section 4). Such constant size assumption driven by supply and demand was used to model the effect of HIV on commercial sex workers and truck drivers using a core group approach in [46,72]. The effect of population variability due to disease induced removal in heterogeneous risk groups is studied comprehensively in $[13,15,60,61,85]$. The total sexually interacting female and male population denoted by $N_{f_{a}}$ and $N_{m_{a}}$ respectively is given by

$$
N_{f_{a}}=S_{f_{a}}+I_{f_{a}}, N_{m_{a}}=S_{m_{a}}+I_{m_{a}} .
$$

We define $c_{i}$, to be the partner acquisition rate for sex group $i$ and $\beta_{i_{j}}$ to be the probability of infection by an individual in sex group $i$ through infectivity channel $j,(j=v, a)$ with subscripts $v$ and $a$ denoting vaginal and anal transmission pathways. Thus $\beta_{i_{v}}$ is the probability of infection by a partner in sex group $i$ through VI and $\beta_{i_{a}}$ is the probability of infection from a partner in sex group $i$ through AI. As per [65, 79] finding's, $\beta_{i_{a}}>\beta_{i_{v}}$. According to [25], the risk of HIV transmission as a result of receptive AI is approximately $3 \%$. For insertive AI the risk is approximately $0.1 \%$. Based on these figures it is obvious that there is much greater risk if the negative partner is receptive in serodiscordant AI. Baggaley [7], assumed that VI and AI are mutually exclusive events per sex act and using this assumption, estimated per partnership transmission probabilities using a Bernoulli process that assumes independence of risk of transmission per sex act within a partnership, equations (A.1) and (A.2). The assumption on mutual exclusivity of VI and AI in any one sex act essentially sets the minimum and maximum per act probabilities of transmission depending on sex act type. If the assumption is dropped and allowance given for both VI and AI to be consummated in any sex act, the true probability must lie between the two extremes. To model HIV transmission risk in heterosexual settings, we assume that on contact, sexual partners may engage in VI only, AI only or they may intermittently switch between the two acts. A parameter $p_{1}\left(0 \leq p_{1} \leq 1\right)$ is defined to be the proportion of infection risk associated with VI and the complement $\left(1-p_{1}\right)$ is the proportion of infection risk associated with AI in a partnership. This proportion depends on exposure to the risk of infection through infecting mode. Exposure risk is impacted upon by many factors including the proportion of the aggregate time spent engaged in VI/AI in unit time, as well as the positioning at the time of ejaculation for the receptive partner. If infecting partner limited themselves to VI in unit time then $p_{1}=1$ and if infecting partner restricted themselves to AI then $p_{1}=0$. The force of infection $\lambda_{i_{j}}$ is dependent on the partner acquisition rate, the infectivity of infecting partner and the proportion of infectious individuals in the opposite sex. The epidemic in Zimbabwe whose data we utilise for model validation, coincided with unprecedented levels of outward migration [77]. The parameter $\alpha$ models the effect of outward migration in the model. The model assumes heterosexual contact only as is mostly common in sub-Saharan Africa. Using the above assumptions, the 
force of infection for the core group is defined as follows:

$$
\left\{\begin{array}{l}
\lambda_{f_{a}}=\left[p_{1} \beta_{m_{v}}+\left(1-p_{1}\right) \beta_{m_{a}}\right] c_{f} v_{m} \frac{I_{m_{a}}}{N_{m_{a}}}, \\
\lambda_{m_{a}}=\left[p_{1} \beta_{f_{v}}+\left(1-p_{1}\right) \beta_{f_{a}}\right] c_{m} v_{f} \frac{I_{f_{a}}}{N_{f_{a}}} .
\end{array}\right.
$$

Using the approach of Tennenbaum et al. [72] to model the group contact constraint, the parameters $v_{f}$ and $v_{m}$ represent the fractions of adult females and males that engage in AI. This implies that the mixing probabilities must satisfy the following law of conservation of sex:

$$
\frac{v_{f} N_{f_{a}}(t)}{c_{m}(t)}=\frac{v_{m} N_{m_{a}}(t)}{c_{f}(t)} .
$$

Equation (2) says that the number of sexual contacts reported by women who engage in AI with men in the core group must equal the number of sexual contacts reported by men who engage in AI with women in the core group. Portrait in Figure 1 depicts the biological system described above.

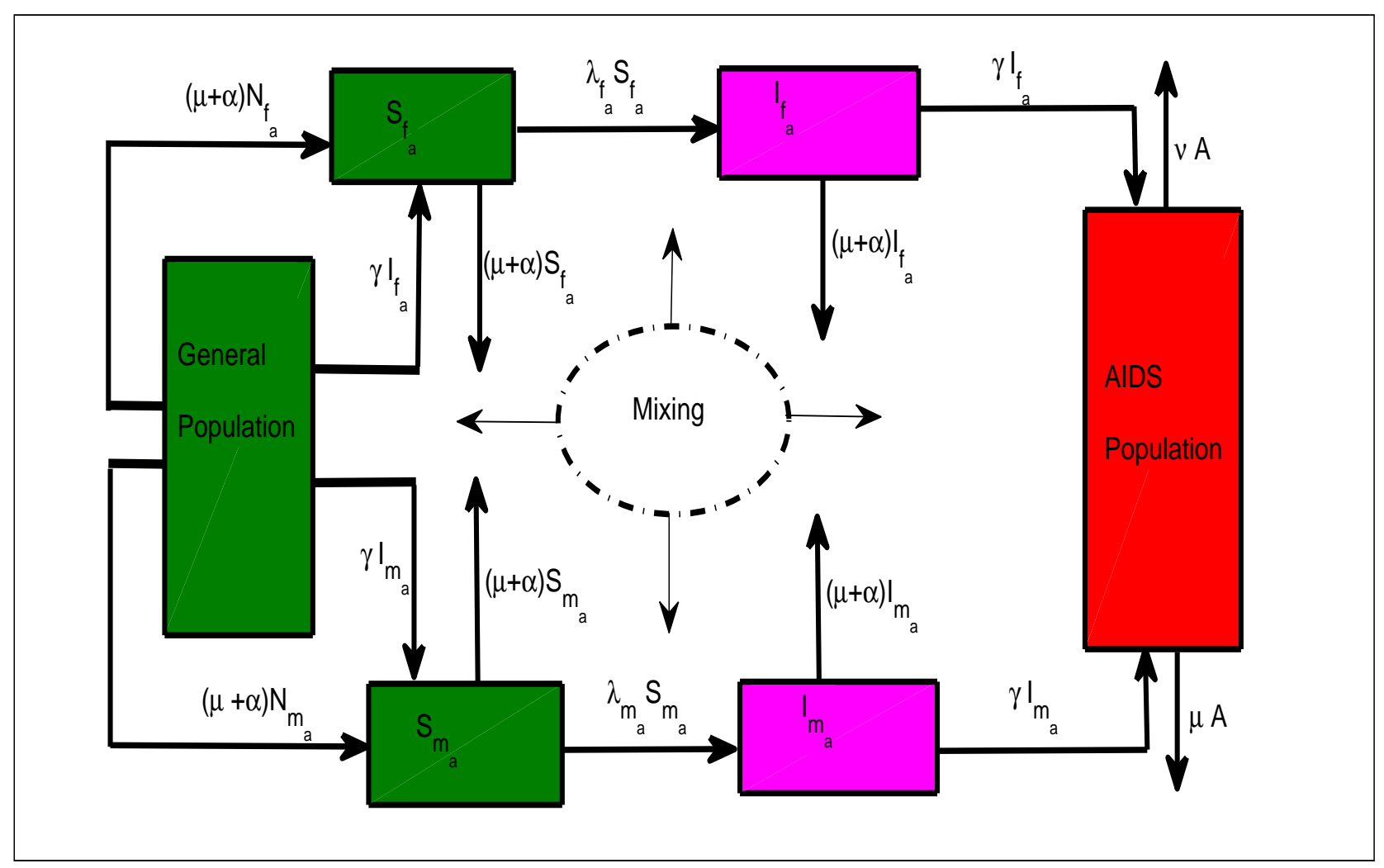

Figure 1: Model flow diagram depicting the biological system representing the core group. The green colour denotes susceptible individuals, pink denotes infected individuals and red denotes individuals who have progressed to AIDS. The white circle represents heterosexual mixing. 
The above formulations and assumptions lead us to the following set of ordinary differential equations,

$$
\begin{aligned}
& \frac{d S_{f_{a}}}{d t}=\mu N_{f_{a}}+\gamma I_{f_{a}}-\left(\mu+\lambda_{f_{a}}\right) S_{f_{a}} \\
& \frac{d I_{f_{a}}}{d t}=\lambda_{f_{a}} S_{f_{a}}-(\gamma+\mu) I_{f_{a}} \\
& \frac{d S_{m_{a}}}{d t}=\mu N_{m_{a}}+\gamma I_{m_{a}}-\left(\mu+\lambda_{m_{a}}\right) S_{m_{a}} \\
& \frac{d I_{m_{a}}}{d t}=\lambda_{m_{a}} S_{m_{a}}-(\gamma+\mu) I_{m_{a}} \\
& \frac{d A}{d t}=\gamma\left(I_{f_{a}}+I_{m_{a}}\right)-(\mu+\nu) A
\end{aligned}
$$

The total interacting female population that engages in both forms of sex is thus given by $N_{f_{a}}=S_{f_{a}}+I_{f_{a}}$ and that of males is given by $N_{m_{a}}=S_{m_{a}}+I_{m_{a}}$. The constant size assumption for the core group allows us to reduce system (3) for the purposes of analysis, to the following system:

$$
\left.\begin{array}{l}
\frac{d I_{f_{a}}}{d t}=\lambda_{f_{a}}\left(N_{f_{a}}-I_{f_{a}}\right)-(\gamma+\mu) I_{f_{a}}, \\
\frac{d I_{m_{a}}}{d t}=\lambda_{m_{a}}\left(N_{m_{a}}-I_{m_{a}}\right)-(\gamma+\mu) I_{m_{a}} .
\end{array}\right\}
$$

\subsection{Basic model properties.}

The state space given by $\mathcal{D}=\left\{\left(I_{f_{a}}, I_{m_{a}}\right) \mid 0 \leq I_{i_{a}} \leq N_{i_{a}}\right\}$ is a rectangular region with vertices

$$
\left\{(0,0),\left(N_{f_{a}}, 0\right),\left(0, N_{m_{a}}\right),\left(N_{f_{a}}, N_{m_{a}}\right)\right\} \text {. }
$$

Using the approach in [46], we analyze the points along the edges of the rectangle as follows:

Points along the horizontal edge on top of $\mathcal{D}$ are of the form $\left(I_{f_{a}}, N_{m_{a}}\right)$ with $0 \leq I_{f_{a}} \leq N_{f_{a}}$. To be well posed, the solution must decrease in the vertical direction of $I_{m_{a}}$. Along the horizontal edge $\left(0, N_{m_{a}}\right) \longleftrightarrow\left(N_{f_{a}}, N_{m_{a}}\right)$, we have $I_{m_{a}}=-(\gamma+\mu) N_{m_{a}}<0$, so that $I_{m_{a}}$ is a decreasing function of time. Biologically, this entails that when all heterosexual males in the practice of AI are infected with HIV, the infected male population will decrease because it cannot grow and exceed the total number of males practicing AI $N_{m_{a}}$. Points on the right edge $\mathcal{D}$ are of the form $\left(N_{f_{a}}, N_{m_{a}}\right)$ where $0 \leq I_{m_{a}}<N_{m_{a}}$. We note that $\dot{I}_{f_{a}}=-(\gamma+\mu) N_{m_{a}}<0$ so that $I_{f_{a}}$ decreases back into the state space. Thus when the number of infected females equals the total number of females, the infected female population will decrease back into the state space. Points on the bottom edge of $\mathcal{D}$ are of the form $\left(N_{f_{a}}, 0\right)$. On that edge, $I_{m_{a}}=\lambda_{m_{a}} N_{m_{a}} \geq 0$, so that if there are no males practicing AI, the population of infected males practicing AI must increase due to infection from infective females practicing AI. On the left edge of $\mathcal{D}$, points are of the form $\left(0, I_{m_{a}}\right)$. On that edge $\dot{I_{f_{a}}}=\lambda_{f_{a}} N_{f_{a}} \geq 0$ meaning that if there are no infected females practicing AI, then the number of infected females practicing AI must increase due to infection by infected males practicing heterosexual AI. This proves positivity and boundedness of solutions. 


\subsection{Disease free equilibrium and reproductive number}

Linearising about the disease free equilibrium $\left(I_{f_{a}}, I_{m_{a}}\right)=(0,0)$, we obtain the Jacobian matrix at the disease free equilibrium

$$
J(0,0)=\left[\begin{array}{cc}
-(\gamma+\alpha+\mu) & \frac{\left(p_{1} \beta_{m_{v}}+\left(1-p_{1}\right) \beta_{m_{a}}\right) c_{f} N_{f_{a}} v_{m}}{N_{m_{a}}} \\
\frac{\left(p_{1} \beta_{f_{v}}+\left(1-p_{1}\right) \beta_{f_{a}}\right) c_{m} N_{m_{a}} v_{f}}{N_{f_{a}}} & -(\gamma+\alpha+\mu)
\end{array}\right] .
$$

Solving the characteristic equation gives the AI induced reproductive number for the core group

$$
\mathcal{R}_{c}=\sqrt{\frac{c_{f} c_{m}\left(\beta_{f_{a}}\left(1-p_{1}\right)+p_{1} \beta_{f_{v}}\right)\left(\beta_{m_{a}}\left(1-p_{1}\right)+p_{1} \beta_{m_{v}}\right) v_{f} v_{m}}{(\gamma+\alpha+\mu)^{2}}} .
$$

The reproductive number is a count of the number of cases generated by an infected individual in his entire infectious lifetime when introduced into a wholly susceptible population. In the absence of AI, $p_{1}=1$ and,

$$
\mathcal{R}_{c}=\sqrt{\frac{c_{f} c_{m} v_{f} v_{m} \beta_{f_{v}} \beta_{m_{v}}}{(\gamma+\alpha+\mu)^{2}}}=\mathcal{R}_{0}
$$

where $\mathcal{R}_{0}$ denotes the basic reproductive number. Since transmission risks from AI exceed those from VI, we can write, $\beta_{f_{a}}=\left(1+\kappa_{1}\right) \beta_{f_{v}}$ and $\beta_{m_{a}}=\left(1+\kappa_{2}\right) \beta_{m_{v}}$ where $\left(\kappa_{1}, \kappa_{2}\right)>0$, and the AI induced reproductive number can be expressed in terms of the basic reproductive number as:

$$
\begin{aligned}
\mathcal{R}_{c} & =\sqrt{\frac{\left(1+\left(1-p_{1}\right) \kappa_{1}\right)\left(1+\left(1-p_{1}\right) \kappa_{2}\right) c_{f} c_{m} \beta_{f_{v}} \beta_{m_{v}} v_{f} v_{m}}{(\gamma+\alpha+\mu)^{2}}} \\
& =\left(1+\left(1-p_{1}\right) \kappa_{1}\right)\left(1+\left(1-p_{1}\right) \kappa_{2}\right) \mathcal{R}_{0},
\end{aligned}
$$

where $\mathcal{R}_{0}$ is defined to be the basic reproductive number for the core group in the absence of AI. Since the coefficient of $\mathcal{R}_{0}$ exceeds unity, clearly, engaging in AI will increase generate higher numbers of new infections and worsen the epidemic. The The following lemma ensures that $J(0,0)$ is stable;

Lemma 1. The disease free equilibrium $\xi_{0}$ exists and is locally stable for $\mathcal{R}_{c}<1$.

\subsection{The endemic equilibrium}

The endemic equilibrium $\xi_{e}$ is given by

$$
\begin{aligned}
\xi_{e} & =\left(I_{f_{a}}, I_{m_{a}}\right) \\
& =\left(\frac{N_{f_{a}}(\gamma+\alpha+\mu)^{2}\left(\mathcal{R}_{c}^{2}-1\right)}{c_{m} v_{f}\left(\beta_{f_{a}}\left(1-p_{1}\right)+p_{1} \beta_{f_{v}}\right)\left(\gamma+\alpha+\mu+c_{f} v_{m}\left(\beta_{m_{a}}\left(1-p_{1}\right)+p_{1} \beta_{m_{v}}\right)\right)},\right. \\
& \left.\frac{N_{m_{a}}(\gamma+\alpha+\mu)^{2}\left(\mathcal{R}_{c}^{2}-1\right)}{c_{f} v_{m}\left(\beta_{m_{a}}\left(1-p_{1}\right)+p_{1} \beta_{m_{v}}\right)\left(\gamma+\alpha+\mu+c_{m} v_{f}\left(\beta_{f_{a}}\left(1-p_{1}\right)+p_{1} \beta_{f_{v}}\right)\right)}\right) .
\end{aligned}
$$

The following result is established

Lemma 2. The endemic equilibrium $\xi_{e}$ exists and is unique for $\mathcal{R}_{c}>1$.

Having established the existence of $\xi_{e}$, we investigate its local stability,

Theorem 1. The endemic steady state $\xi_{e}$ of system (3) is locally asymptotically stable if $\mathcal{R}_{c}>1$. 
Proof. The Jacobian matrix system (3) evaluated at the endemic equilibrium is given by $J\left(\xi_{e}\right)$ where

$$
J\left(\xi_{e}\right)=\left[\begin{array}{cc}
-\epsilon_{1}-\frac{\tau_{f}\left(\mathcal{R}_{c}^{2}-1\right)}{\left(\epsilon_{1}+c_{m} \tau_{f} v_{f} \beta_{f}\right)} & \frac{c_{f} N_{f_{c}} \tau_{m} v_{m} \beta_{m}}{N_{m_{c}}}\left(1+\frac{\epsilon_{1}^{2}\left(\mathcal{R}_{c}^{2}-1\right)}{c_{m} \tau_{f} v_{f} \beta_{f} \hat{\phi}}\right. \\
\frac{c_{m} N_{m_{c}} \tau_{f} v_{f} \beta_{f}}{N_{f_{c}}}\left(1+\frac{\epsilon_{1}^{2}\left(\mathcal{R}_{c}^{2}-1\right)}{c_{f} \tau_{f} v_{m} \beta_{m} \tau^{* *}}\right. & -\epsilon_{1}-\frac{\epsilon_{1}^{2}\left(\mathcal{R}_{c}^{2}-1\right)}{\tau^{*}}
\end{array}\right],
$$

where $\epsilon_{1}=(\gamma+\alpha+\mu), \tau^{*}=\left(\epsilon_{1}+c_{f}\left(\tau_{m} v_{m} \beta_{m}\right)\right), \tau^{* *}=\left(\epsilon_{1}+c_{m} \tau_{m} v_{f} \beta_{f}\right), \tau_{f}=\beta_{f_{a}}\left(1-p_{1}\right)+p_{1} \beta_{f_{v}}, \hat{\phi}=$ $\left(\epsilon \kappa_{1}+c_{f} \tau_{m} v_{m} \beta_{m}\right)$ and $\tau_{m}=\beta_{m_{a}}\left(1-p_{1}\right)+p_{1} \beta_{m_{v}}$. The trace of $J\left(\xi_{e}\right)$ is given by

$$
\begin{aligned}
\operatorname{tr}\left(J\left(\xi_{e}\right)\right) & =-s^{*}\left(\frac{\left(\mathcal{R}_{c}^{2}-1\right)}{\left(\phi^{*}+\phi^{* *}\left(\beta_{f_{a}}\left(1-p_{1}\right)+p_{1} \beta_{f_{v}}\right)\right.}+\frac{\left(\mathcal{R}_{c}^{2}-1\right)}{\left(\phi^{*}+\phi^{* * *}\left(\beta_{m_{a}}\left(1-p_{1}\right)+p_{1} \beta_{m_{v}}\right)\right.}\right) \\
& <0,
\end{aligned}
$$

where $\phi^{*}=(\gamma+\mu), \phi^{* *}=c_{m} v_{f}, s *=\phi^{*}\left(2+\phi^{*}\right)$ and $\phi^{* * *}=c_{f} v_{m}$. The determinant of $J\left(\xi_{e}\right)$ is given by

$$
\operatorname{det}\left(J\left(\xi_{e}\right)\right)=(\gamma+\mu)^{2}\left(\mathcal{R}_{c}^{2}-1\right)>0 .
$$

Thus the eigenvalues of the Jacobean matrix $\left(J \xi_{e}\right)$ have negative real parts. This implies that $\xi_{e}$ is asymptotically stable whenever it exists. This completes the proof.

\subsection{Global stability of equilibria}

We have shown that the solutions to model system (3) are bounded and approach an equilibrium $\xi_{0}$ if $\mathcal{R}_{c}<$ 1 and $\xi_{e}$ if $\mathcal{R}_{c}>1$. In order to prove global stability of equilibria using Bendixson-Dulac Criterion [14], we need to rule out the existence of periodic solutions.

Lemma 3. If $\mathcal{R}_{c}<1$, the disease free equilibrium $\xi_{0}$ is globally asymptotically stable in the interior of $\mathcal{D}$, whereas if $\mathcal{R}_{c}>1, \xi_{0}$ if it exists, is a saddle and the endemic equilibrium state $\xi_{e}$ of system (3) is globally asymptotically stable in the interior of $\mathcal{D}$.

Proof. Consider the dynamical system

$$
\begin{aligned}
\frac{d I_{f_{a}}}{d t} & =F\left(I_{f_{a}}, I_{m_{a}}\right), \\
\frac{d I_{m_{a}}}{d t} & =G\left(I_{f_{a}}, I_{m_{a}}\right),
\end{aligned}
$$

where $I_{f_{a}}$ and $I_{m_{a}}$ are continuously differentiable functions on some simply connected domain $\mathcal{D} \subset \mathbb{R}^{2}$. If $\nabla .(F, G)=\frac{\partial F}{\partial I_{f_{a}}}+\frac{\partial G}{\partial I_{m_{a}}}$ is of one sign in $\mathcal{D}$, there cannot be a closed orbit within $\mathcal{D}$. From system (3)

$$
\frac{\partial F}{\partial I_{f_{a}}}+\frac{\partial G}{\partial I_{m_{a}}}=-2(\gamma+\mu)-\left[\frac{c_{f} I_{m_{a}} v_{m}\left(\beta_{m_{a}}\left(1-p_{1}\right)+p_{1} \beta_{m}\right)}{N_{m_{a}}}+\frac{c_{m} I_{f_{c}} v_{f}\left(\beta_{f_{a}}\left(1-p_{1}\right)+p_{1} \beta_{f}\right)}{N_{f_{a}}}\right]<0
$$

which is always negative and we conclude that there are no limit cycles in $\mathcal{D}$. Since the model is well posed (solution exists and is unique), has no periodic orbits and tends to some equilibrium, then the disease free and endemic equilibrium when they exist are globally stable save for $\xi_{0}$ which is a saddle if it exists for $\mathcal{R}_{c}>1$. This completes the proof. 


\section{General population model}

In general many countries in Sub-Saharan Africa for example Zimbabwe, began publishing national HIV sentinel data in 1990. Presently, there is no published data on HIV prevalence in sub-groups that engage in heterosexual AI in the region. HIV information that is readily available relates to prevalence in the overall population. We dispense with the constant population size assumption in Section 2 and make the core group size proportional to the variable sized sexually active general population. Althouh most studies of heterogeneous groups pressume that mixing is assortative [], the paucity of data on AI in Zimbabwe makes it difficult to quantify such a relationship. In this study we thus assume random mixing for tractibility. By assuming that the population is well mixed, and that members of the core group have partners in the general population, we can fit and parameterise the model using prevalence data published by UNAIDS [75]. The core group size is treated as a variable fit in order to explore the parameter space related to heterosexual AI. We assume the general population to be naive of $\mathrm{AI}$ and therefore only penile-vaginal intercourse occurs on interaction with individuals in the core group. Let $\Lambda$ denote the general population recruitment rate, then the female susceptible general population $S_{f_{v}}$ is replenished at a constant rate $\pi_{0}$ with the complement $\left(1-\pi_{0}\right)$ going into the male susceptible general population $S_{m_{v}}$. On effective contact with the HIV virus, susceptible individuals in the general population move into the infectious classes $I_{f_{v}}$ and $I_{m_{v}}$ at a time dependent force of infection $\lambda_{i_{v}}$. The parameter $\psi$ models the proportion of the general population that is initiated into the core group. Variables and parameters related to the core group remain unchanged.

Using the above assumptions the following forces of infection for the sub-groups are defined,

$$
\left.\begin{array}{rl}
\lambda_{f_{v}}= & \frac{c_{f} \beta_{m_{v}}\left[I_{m_{v}}+I_{m_{a}}\right]}{S_{m_{v}}+I_{m_{v}}+N_{m_{a}}}, \\
\lambda_{f_{a}}= & \frac{c_{f}\left[\beta_{m_{v}} I_{m_{v}}+\left(p_{1} \beta_{m_{v}}+\left(1-p_{1}\right) \beta_{m_{a}}\right) I_{m_{a}}\right]}{S_{m_{v}}+I_{m_{v}}+N_{m_{a}}}, \\
\lambda_{m_{v}}= & \frac{c_{m} \beta_{f_{v}}\left[I_{f_{v}}+I_{f_{a}}\right]}{S_{f_{v}}+I_{f_{v}}+N_{f_{a}}}, \\
\lambda_{m_{a}}= & \frac{c_{m}\left[\beta_{f_{v}} I_{f_{v}}+\left(p_{1} \beta_{f_{v}}+\left(1-p_{1}\right) \beta_{f_{a}}\right) I_{f_{a}}\right]}{S_{f_{v}}+I_{f_{v}}+N_{f_{a}}} .
\end{array}\right\}
$$

In 1985 when the first HIV/AIDS case was reported in Zimbabwe, it is estimated that around 10\% of the adult population was infected with HIV [73]. Prevalence rose sharply in the late 1980s and early 1990s, and is thought to have peaked at above $25 \%$ around 1997 [56]. There after it seems to have plateaued off with AIDS related deaths balancing off new infections. Zimbabwe became the first Southern African country to record a significant drop in the HIV prevalence rate in the adult age group (15-49) from $24.6 \%$ in 2003 to $20.1 \%$ in 2005 [76] and subsequently declining to $14.3 \%$ in 2010 [74]. Possible causes include changes in sexual behaviour due to experiential learning. As more people become symptomatic and visible to society, at risk individuals tend to acquire greater HIV awareness. Thus increased condom uptake and partnerships reduction has been observed since 2005 [73]. This behaviour change must be included in the data fit by making the force of infection in equation (1) responsive to this reduced susceptibility. Let the probability of infection be a function of the symptomatic rate $(\gamma)$ so that the contact rate function is modelled by

$$
c_{i} \beta_{i_{j}} e^{-\varpi \gamma P}
$$


where the parameter $\varpi$ is the response due to reducing susceptibility resulting from people seeing individuals develop AIDS symptoms and $P=\frac{I_{i}}{S_{i}+I_{i}}$ is the prevalence function. Such behavioural change function has been used before by $[28,83,84]$. The model flow diagram is depicted in Figure 2 .

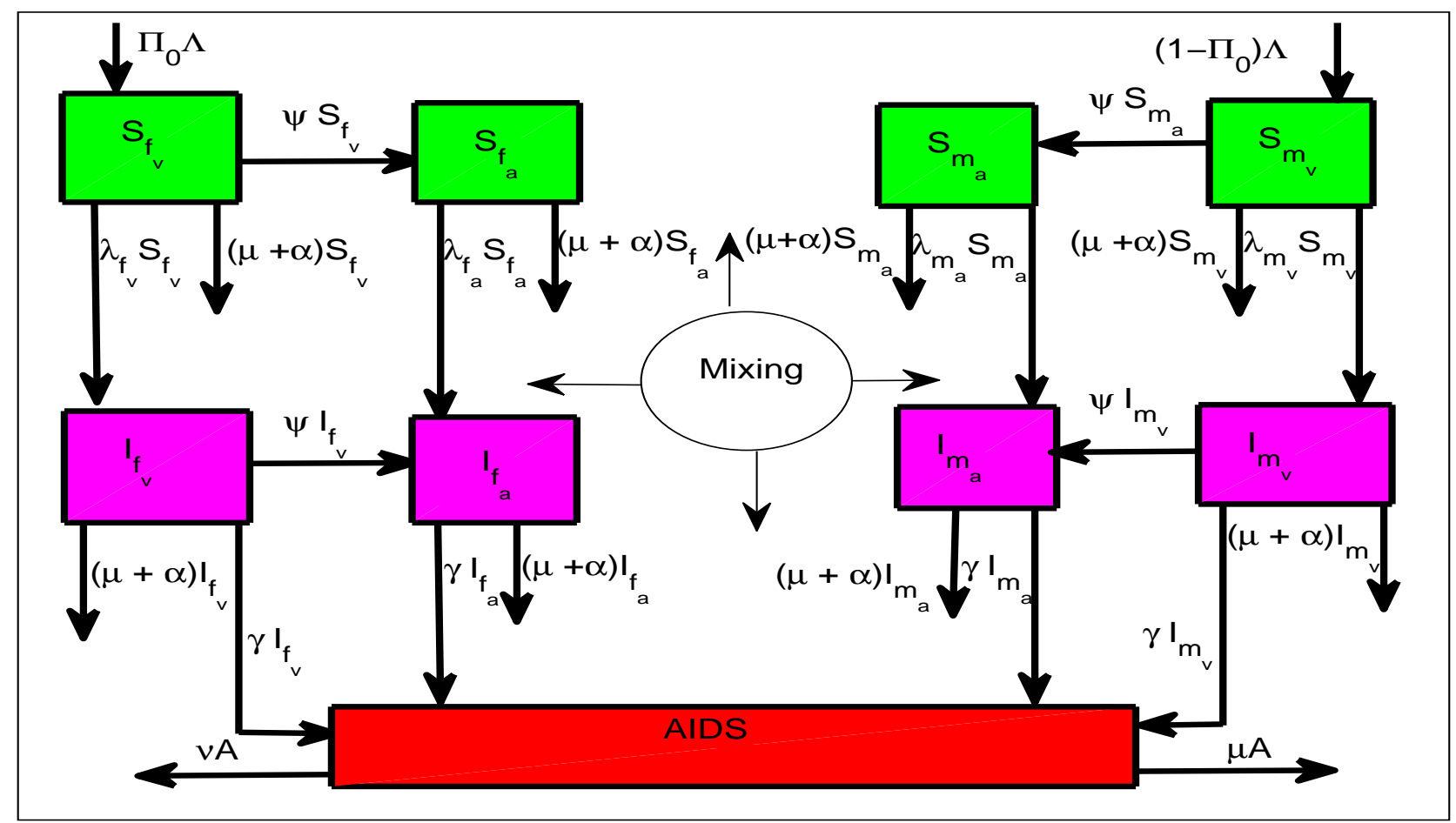

Figure 2: Model flow diagram representing biological system with the core grouped coupled to the general population. The green color denotes susceptible individuals, pink denotes infected individuals and red denotes individuals who have progressed to AIDS. The white circle represents heterosexual mixing.

Combining the above assumptions, the biological system transforms into the following set of deterministic ordinary differential equations: 


$$
\begin{aligned}
& \frac{d S_{f_{v}}}{d t}=\pi_{0} \Lambda-\left(\mu+\alpha+\psi+\lambda_{f_{v}}\right) S_{f_{v}} \\
& \frac{d I_{f_{v}}}{d t}=\lambda_{f_{v}} S_{f_{v}}-(\mu+\alpha+\psi+\gamma) I_{f_{v}} \\
& \frac{d S_{m_{v}}}{d t}=\left(1-\pi_{0}\right) \Lambda-\left(\mu+\alpha+\psi+\lambda_{m_{v}}\right) S_{m_{v}} \\
& \frac{d I_{m_{v}}}{d t}=\lambda_{m_{v}} S_{m_{v}}-(\mu+\alpha+\psi+\gamma) I_{m_{v}} \\
& \frac{d S_{f_{a}}}{d t}=\psi S_{f_{v}}-\left(\mu+\alpha+\lambda_{f_{a}}\right) S_{f_{a}} \\
& \frac{d I_{f_{a}}}{d t}=\psi I_{f_{v}}+\lambda_{f_{a}} S_{f_{a}}-(\mu+\alpha+\gamma) I_{f_{a}} \\
& \frac{d S_{m_{a}}}{d t}=\psi S_{m_{v}}-\left(\mu+\alpha+\lambda_{m_{a}}\right) S_{m_{a}} \\
& \frac{d I_{m_{a}}}{d t}=\psi I_{m_{v}}+\lambda_{m_{a}} S_{m_{a}}-(\mu+\alpha+\gamma) I_{m_{a}} \\
& \frac{d A}{d t}=\gamma\left(I_{f_{v}}+I_{f_{a}}+I_{m_{v}}+I_{m_{a}}\right)-(\nu+\mu) A
\end{aligned}
$$

The usual properties of well posedness of the model that include positivity and invariance of solutions follow using similar approaches described above in Section 2.1. The goal here is to explore the parameter space for the combined model and to fit the model to HIV prevalence data for Zimbabwe.

\section{Numerical analysis and results}

Parameter base values from studies in sub-Saharan Africa were used in this study to explore the qualitative and quantitative features of the model through simulations. The key components of an HIV transmission model are the transmission rates per partnership and the number of partnerships. Transmission probabilities per partner are dependent on the average number of contacts per partner and the mean probability of transmission per contact [35]. The mean number of partnerships in sub-Saharan Africa is very difficult to predict but HIV has been shown to spread even in populations reporting low partnership acquisition rates [36]. Results in [10] showed the proportion of men reporting that they have had 2 or more sex partners in a year ranging from $1 \%$ to $28 \%$ in sub-Saharan Africa. In [71] the mean number of lifetime sexual partners ranged from 3.4 to 12.9 with a mean of 6.3 in sexually active men in the region. Mean lifetime number of partners of men in the immediate local community was predictive of hazard of HIV acquisition in women. In this study, the baseline mean number of partners is taken to be 3 but allowed to vary between 1 and 13 partners per year in the parameterisation of the model.

Heterosexual VI transmission risk is estimated at very low probabilities [30], but varies from and within regions. Varghese et al. [79], estimate the range for insertive and receptive VI to be 5-10 infections per 10000 exposures. A study in Rakai [81] showed average transmission rate per coital act starting off at a peak of 0.0082 within the first 2.5 months of seroconversion, falling to $0.0007 /$ per coital act 15 months after seroconversion and rising to $0.0028,6$ to 25 months before the death of the index partner. A similar 
study by [29] showed transmission per coital act averaging 1 per 1000. The per-contact probability of HIV transmission for receptive unprotected AI was estimated to be $1.43 \%$ while that for insertive unprotected AI was estimated at $0.62 \%$ [37]. Varghese et al. [79] puts the risk of transmission for receptive AI at 50 per 10000 exposures and that for insertive AI at 6.5 per 10000. Baggaley et al. Baggaley et al. [7] estimated the risk of receptive AI at $1.4 \%$ but did not provide an estimate for insertive AI. Using a similar approach to that in $[35,36]$, that $\zeta_{i_{j}}$ is the per act probability of the risk of an infective in group $i$ infecting their partner through infectivity route $j$, the probability that a susceptible in group $j$ will avoid infection in a contact is $\left(1-\zeta_{i_{j}}\right)$. Thus in $n$ contacts, the probability that a susceptible will avoid infection with an infective partner is $\left(1-\zeta_{i_{j}}\right)^{n}$ and the probability that the susceptible will fail to avoid infection in $n$ contacts with a partner, that is, the per partnership probability of transmission $\beta_{i_{j}}$ is given by

$$
\beta_{i_{j}}=1-\left(1-\zeta_{i_{j}}\right)^{n} .
$$

While there is no known relationship between the number of partners $c_{i}$ and the number of contacts per partner $n$, it is reasonable to assume that $n$ decreases with increasing $c_{i}$, that is individuals with numerous partners have lesser contacts per partner than individuals with fewer partners. In earlier studies, [35,36] and later [58] used a simple function with such property to be $n=\frac{104}{c_{i}}+1$. Thus $\beta_{i_{j}}=1-\left(1-\zeta_{i_{j}}\right)^{\frac{104}{c_{i}}}+1$. A study of monogamous heterosexual couples in Rakai, Uganda, showed that the mean frequency of intercourse was 8.9 per month, declining with age and HIV-1 viral load of index partner [29]. As in $[35,36]$, we assume that people with few partners have 2 contacts per week and people with many partners have slightly more than one contact per partner. Using highest estimates by [68] for insertive and receptive penal -vaginal transmission that is 0.0014 and 0.005 respectively and using estimates by [37] for insertive and receptive anal transmissions $0.62 \%$ and $1.43 \%$ respectively we obtain the following per partner transmission probabilities which are independent and exclusive to route of transmission and use these as the baseline values:

$$
\beta_{f_{v}}=0.0487, \beta_{m_{v}}=0.1637, \beta_{f_{a}}=0.1989 \text { and } \beta_{m_{a}}=0.4017 .
$$

We assumed that VI and AI can occur in a single sex act and with independent risk of transmission and introduced a parameter $p_{1}$ which models the proportion of infection risk associated with VI in a partnership in unit time. The complement models the exposure to infection from AI. The dynamics governing AI have not been studied comprehensively and remain mystique. Thus the value of $p_{1}$ that we use is arbitrary and is allowed to vary between 0 and 1 . The remainder of the parameters and their sources are shown in Table 4 including fitted values. 


\begin{tabular}{|c|c|c|c|c|}
\hline Symbol & $\begin{array}{l}\text { Parameter definition } \\
\text { baseline } \\
\text { value }\end{array}$ & Range or & $\begin{array}{l}\text { Source of data } \\
\text { value }\end{array}$ & Fitted \\
\hline & $\begin{array}{l}\text { Biological, epidemiological and } \\
\text { demographic parameters }\end{array}$ & & & \\
\hline$\mu$ & Natural death rate & 0.02 & (Mukandavire et al., 2009) & - \\
\hline$\Lambda$ & Newly sexually matured individuals & $52600 / \mathrm{yr}$ & (Mukandavire et al., 2009) & - \\
\hline$\phi$ & Proportion of new recruits who are female & variable & - & - \\
\hline$\left(\zeta_{f_{v}}, \zeta_{m_{v}}\right)$ & $\begin{array}{l}\text { Per vaginal coital act probability of HIV } \\
\text { transmission }\end{array}$ & {$[0.0005,0.0010]$} & {$[79,81]$} & - \\
\hline$\left(\beta_{f_{v}}, \beta_{m_{v}}\right)$ & $\begin{array}{l}\text { Per partner probability } \\
\text { of vaginal HIV transmission }\end{array}$ & {$[0.0108,0.0215]$} & derived & $(0.1073,0.1068)$ \\
\hline$\gamma$ & Average incubation period & 0.125 & (Mukandavire et al., 2009) & \\
\hline$\left(c_{f}, c_{m}\right)$ & Frequency of partner acquisition & $\begin{array}{l}5[1-13] \\
\text { partners/yr }\end{array}$ & $\begin{array}{l}{[10]} \\
{[71]}\end{array}$ & \\
\hline$\nu$ & AIDS induced death rate & 0.333 & {$[59]$} & \\
\hline$\alpha$ & Emigration rate & 0.1491 & (derived & \\
\hline \multirow[t]{2}{*}{$\varpi$} & Response parameter for behaviour change & $>0$ & - & 8.05 \\
\hline & $\begin{array}{l}\text { AI related } \\
\text { transmission parameters }\end{array}$ & & & \\
\hline$\left(\zeta_{f_{a}}, \zeta_{m_{a}}\right)$ & $\begin{array}{l}\text { Per anal coital act probability } \\
\text { of HIV transmission }\end{array}$ & {$[0.0062,0.0143]$} & $\begin{array}{l}{[6]} \\
{[37]} \\
{[48]}\end{array}$ & - \\
\hline$\left(\beta_{f_{a}}, \beta_{m_{a}}\right)$ & $\begin{array}{l}\text { Per partner probability } \\
\text { of HIV transmission via AI }\end{array}$ & {$[0.1989,0.4017]$} & derived & $0.2950,0.2947$ \\
\hline$p_{1}$ & Proportion of infection risk associated with VI & $0-1$ & variable & 0.0913 \\
\hline$\psi$ & Variable core group proportion size & $0-1$ & variable & 0.0720 \\
\hline
\end{tabular}

Table 2: Model parameters and their interpretation. The abbreviations AI and VI refer to anal and vaginal intercourse respectively. 


\title{
4.1 Uncertainty and sensitivity analysis of model parameters
}

We determine parameter sensitivity qualitatively through plots of input against output values, as a preliminary and then quantitatively through computation of the partial rank correlation coefficients (PRCC). We used scatter plots to visually determine the degree of correlation between the model output and model parameters at a discrete time point $(\mathrm{t}=20)$ at the end of our modelling period. The scatter plots are shown in Figure 3. Figures 3(b) and 3(f) give a clear visual of the strong relationship between the model output and the parameters denoting core group size and emigration. A weak monotonic correlation between the input parameters and the dependent variable is observed in Figures 3(a), 3(c), 3(d) and 3(e) but the visualisation of a lack of a strong relationship may be due to scaling. Thus preliminary results using scatter plots indicate that an increase in the proportion of individuals engaging in AI will result in an increase in epidemic levels whereas outward migration would have the opposite effect.

\begin{abstract}
Above each plot is the PRCC index and the $p$-value. Partial rank correlation describes the relationship between two variables while simultaneously removing the effects of several other variables, from the relationship. The $p$-value quantifies the level of uncertainty an LHS parameter contributes to the model. Partial rank correlation helps focus data collection on the most sensitive parameters allowing for improved reliability in model output. Parameters that are shown to be insignificant can be removed from the model to give the modeller more degrees of freedom.
\end{abstract}




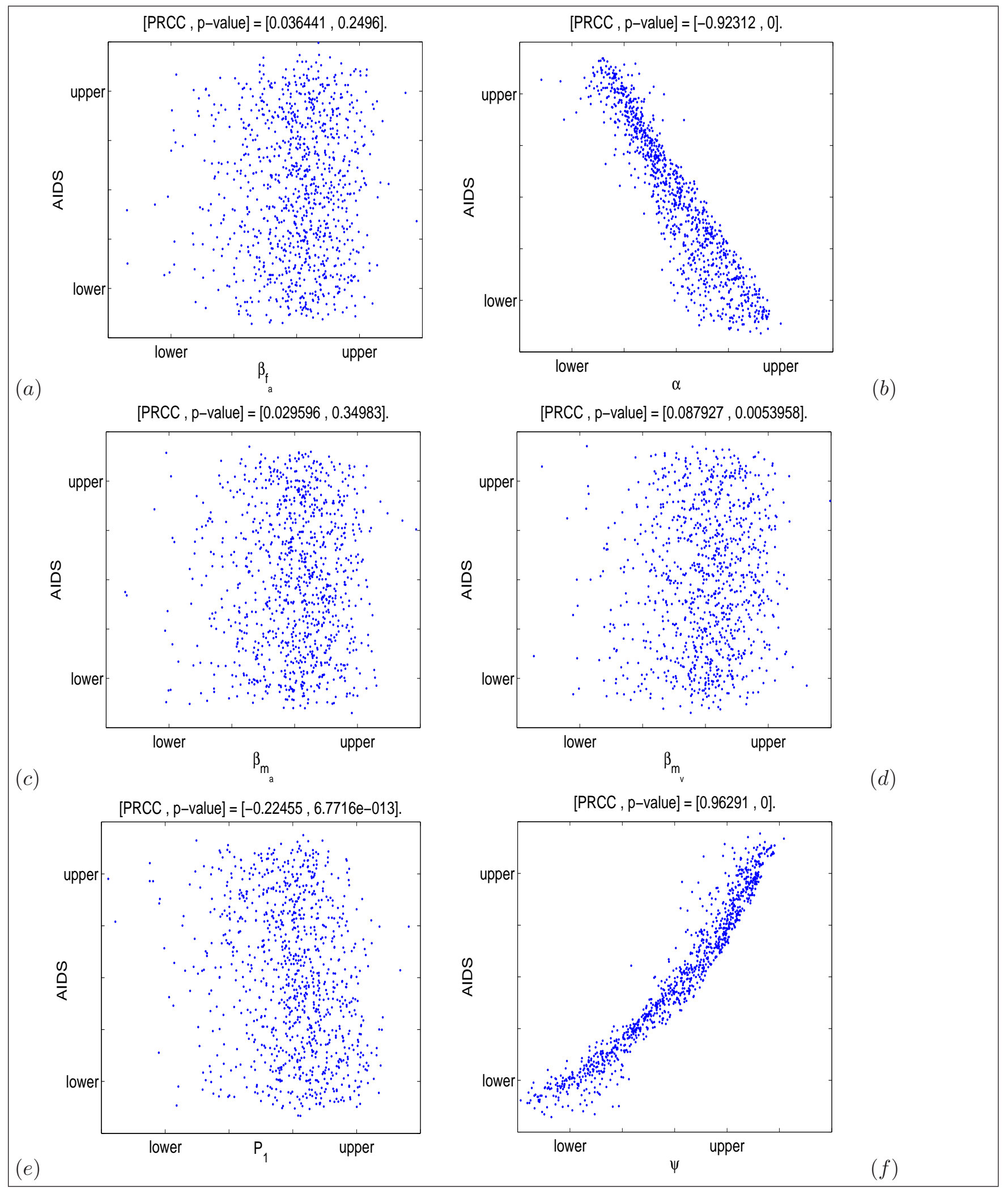

Figure 3: Scatter plots illustrating the relationship between the AIDS population (A), and model parameters examined at time step $(t=20)$. The baseline parameter values are given in Table 4 .

Figure 4 is a graphical illustration of the quantitative relationship between the influential 
input parameters and the number of AIDS cases. Parameters with positive PRCCs will result in an increase in the number of AIDS cases when increased while an increase in parameters with negative PRCCs will result in reduced number of AIDS cases.

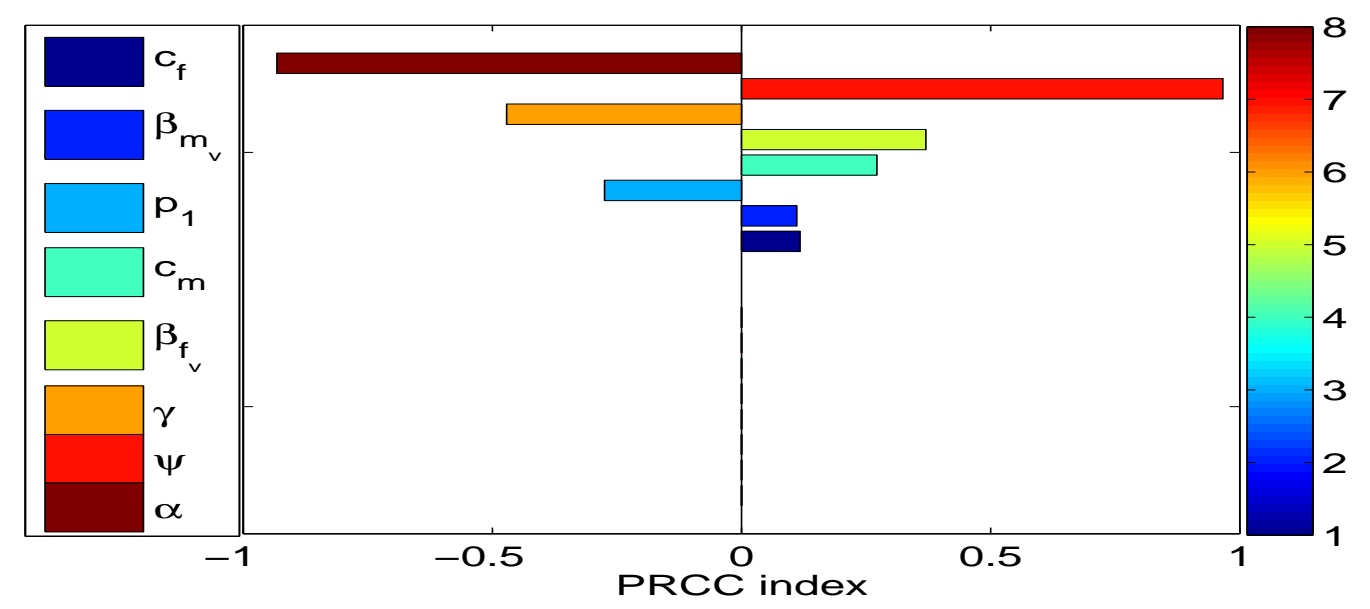

Figure 4: PRCC indices illustrating the sensitivity of the AIDS population size to changes in model parameters at time step $t=20$.

Figure 4 shows that the most influential parameters in the model include the variable core group size, the emigration rate, the proportion of infection risk associated with VI and both the partnership rates as well as the per partnership transmission rates for the general population. Across the epidemic span, relative influence of parameters remains the same. Table 3 is a summary of PRCC indices for both VI and heterosexual AI when data on the epidemic first became available (start), at the peak of the epidemic and at the end of our modelling period (2010).

\begin{tabular}{l|cc|lc|lc}
\hline Epidemic phase & & Start & & Peak & \multicolumn{2}{c}{ End } \\
\hline Parameter & PRCC & $p$-value & PRCC & $p$-value & PRCC & $p$-value \\
\hline$\psi$ & 0.9990 & $<0.001$ & 0.97911 & $<0.001$ & 0.96467 & $<0.001$ \\
$p_{1}$ & -0.0784 & $<0.001$ & -0.3888 & $<0.001$ & -0.2515 & $<0.001$ \\
$c_{f}$ & 0.2936 & $<0.001$ & 0.595 & $<0.001$ & 0.071488 & $<0.001$ \\
$c_{m}$ & 0.7980 & $<0.001$ & 0.72696 & $<0.001$ & 0.27271 & $<0.001$ \\
$\beta_{m_{v}}$ & 0.2177 & $<0.001$ & 0.4665 & $<0.001$ & 0.0429 & $<0.001$ \\
$\beta_{f_{v}}$ & 0.9148 & $<0.001$ & 0.8626 & $<0.001$ & 0.3707 & $<0.001$ \\
$\beta_{m_{a}}$ & 0.0052 & $>0.05$ & 0.0044 & $>0.05$ & 0.035 & $>0.05$ \\
$\beta_{f_{a}}$ & 0.0051 & $>0.05$ & 0.0755 & $>0.05$ & 0.0750 & $>0.05$ \\
\hline
\end{tabular}

Table 3: The PRCCs are between the input parameter and the output values (total AIDS cases). The qualitative relationship between the input parameter and the model output is shown by the - and + signs.

Figure 5 is a graphic illustration of the relative influence of the parameters over the epidemic span. From Table 3 and Figure 5, the variable core group size, partnership rates, the per partnership transmission rates for the general population are most deterministic of the AIDS population size generated by the model. Per partnership probability of transmission rates related to heterosexual AI are less influential through out the epidemic. Over time, as the epidemic recedes, parameter influence reduces. 


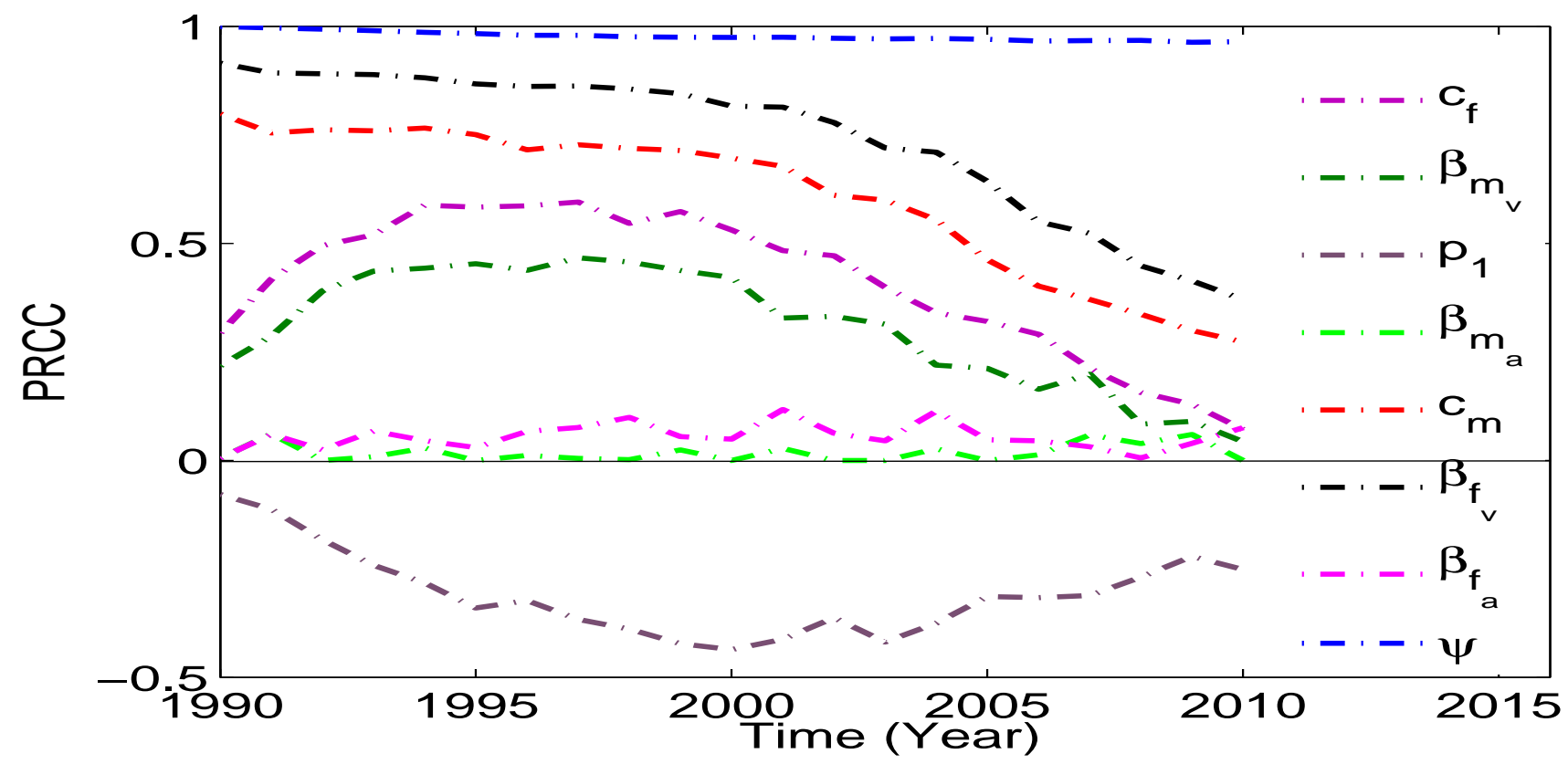

Figure 5: PRCC indices illustrating the sensitivity of the AIDS population size to changes in model parameters throughout the epidemic.

\subsection{Model fit to epidemic and projections}

To parameterise the model, it is fit using least squares curve fitting function in Matlab, across the epidemic span starting from the first year when data from sentinel observations was first made available [75]. To obtain a global solution to the fit, the multi-start function was employed to produce parameter values that gave the best model fit to the data. These parameters were then used to make projections to the year 2020. The size of the core group was treated as a fit variable to obtain the size most consistent with reported data [75]. At the 95\% level of confidence, the core group size estimate ranges between $5-20 \%$, a figure higher than results from observational studies $[29,53]$ within sub-Saharan Africa. In order to assess the potential of an increase in the core group size and the prevalence of heterosexual AI, the core group size was hypothetically increased to $20 \%$ in $\mathbf{2 0 1 0}$ to coincide with reported prevalence of AI outside of Africa $[29,53]$ and the subsequent upward shift in the epidemic was qualified to be the potential impact of increased prevalence of heterosexual AI in high HIV prevalence settings. Using bootstrapping technique in MATLAB, the $95 \%$ credible intervals were computed for the fitted parameters and are shown in Table 4.

\begin{tabular}{|l|c|c|c|}
\hline Parameter & $\beta_{m_{v}}$ & $\beta_{f_{v}}$ & $\beta_{f_{a}}$ \\
\hline Estimate & $0.1068[0.1049-0.1291]$ & $0.1073[0.0823-0.1077]$ & $0.2950[0.1575-0.3272]$ \\
\hline Parameter & $\beta_{m_{a}}$ & $p_{1}$ & $\psi$ \\
\hline Estimate & $0.2947[0.1539-0.3497]$ & $0.0913[0.09134-0.1547]$ & $0.0720[0.0562-0.2029]$ \\
\hline
\end{tabular}

Table 4: The 95\% credible intervals for each input parameter.

We used the best fit from bootstrapping to plot the figures in Figure 6. Figure 6(a) depicts the model fit to HIV prevalence data across the epidemic. Figure 6(c) is a projection of prevalence to calender year 2020 and shows HIV remaining endemic in the population through the decade at a stable prevalence rate of around $14 \%$. Together, prevalence and the 
number of people living with HIV index allow for forward planning. However, it is more informative to obtain the actual number of new infections per unit time. Figure 6(b) is a derivative of HIV incidence from the model fit to prevalence data and Figure 6(d) is an extrapolation of HIV incidence to year 2020. Figure 6(e) shows the number of people living with HIV and Figure 6(f) is the predicted number of people living with HIV through the next decade. 


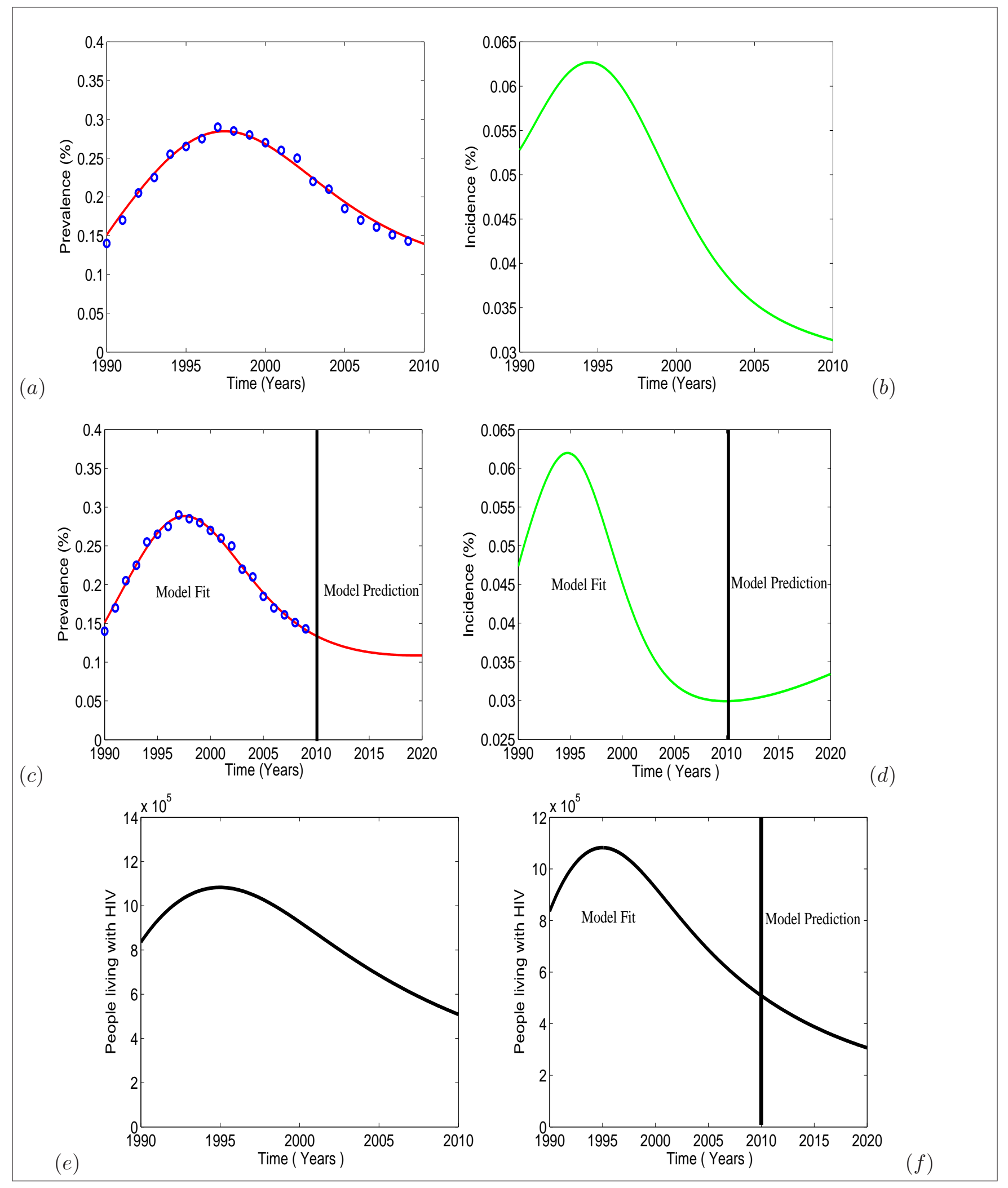

Figure 6: (a) Model fit to UNAIDS prevalence data for Zimbabwe. (b) Incidence curve corresponding to the prevalence from the model fit to UNAIDS data for Zimbabwe. (c) HIV model projection of prevalence until 2020. (d) HIV model projection of incidence till year 2020. (e) Number of people living with HIV from the model fit. $(f)$ Projected number of people living 2020. planning. Data is obtained from [75] and parameter values giving the best fit are given in Table 4. 
While the data at hand is non-risk specific, in Figure 7, we split the new infections according to their source of infection. From Figure $7(\mathrm{a})$ and Figure $7(\mathrm{~b})$, incidence due to VI ranged from $0.02-0.045 \%$ and $0.005-0.027 \%$ from heterosexual AI. The HIV infection risks associated with AI are recognised and inform HIV policying for men who have sex with men, yet the same risks are ignored in HIV policing for heterosexuals. By quantifying and apportioning the incidence due to VI and heterosexual AI risks, we put emphasis on the necessity for public health practitioners to target the specific multiple infection pathways rather than use the "one treats all" approach to intervention.

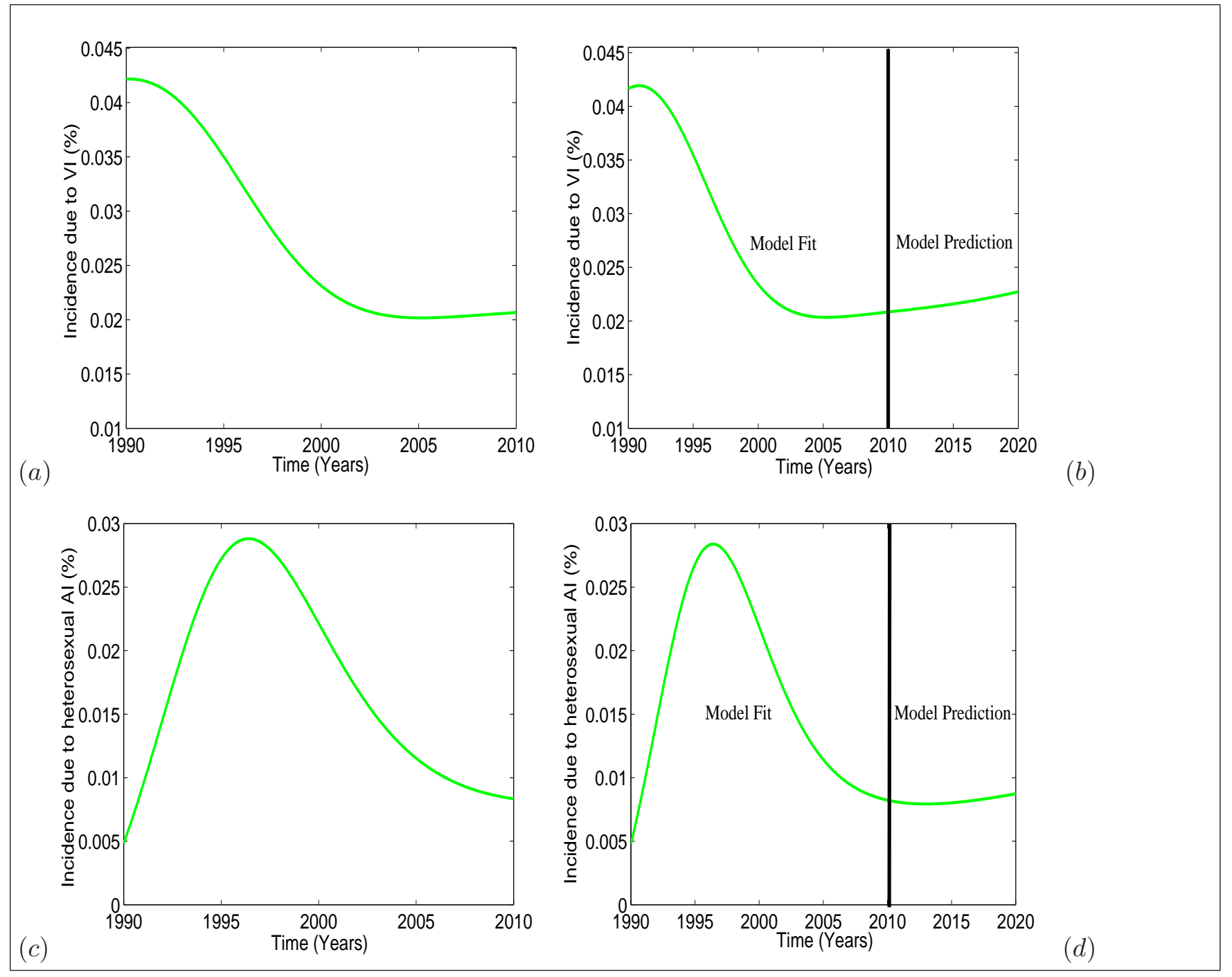

Figure 7: (a) Incidence of new infections due to VI. (b) Projected incidence of new infections due to VI. (c) Incidence of new infections due to heterosexual AI. $(d)$ Projected incidence of new infections due to heterosexual AI.

The core group engaging in heterosexual AI outside of Africa is thought to average 20\%, [29, 53]. This high prevalence of heterosexual AI is happening in low HIV prevalence settings. Moreover, the size of the core group is shown in the sensitivity analysis, to be highly deterministic of the epidemic size. Thus an assessment of the potential impact of an increase in the core group size in high HIV prevalence settings is a necessity. We set the size of the core group in the calendar year 2010 to $20 \%$ and allowed the proportion of infection risk 
associated with AI to vary, starting from a baseline of zero exposure $\left(p_{1}=1\right)$. When exposure to infection via the anal pathway is increased to $50 \%$, from baseline, HIV prevalence would escalate by $23 \%$ from the baseline values in the year 2020. If exposure risk to heterosexual AI is increased to $70 \%$, HIV prevalence rate will escalate by $38 \%$ from baseline values in year 2020. Thus the effect of the core group on overall HIV dynamics is amplified in settings with higher HIV prevalence. Figure 8(c) is a plot of the AI induced reproductive number $\mathcal{R}_{A}$ against $p_{1}$ the exposure to the risk of penile-vaginal infection. The graph shows an inverse relationship between $\mathcal{R}_{A}$ and $p_{1}$ implying that reducing exposure to the competing risk of infection due to AI would reduce the epidemic. However, for the kind of epidemic experienced in Zimbabwe, curtailing AI is not sufficient to control the epidemic (Figures 6(a), 6(b), 6(c), 6(d), 7(a), 7(b)), 8(a) and 8(b)) and other intervention measures targeted at the general population are necessary given the sensitivity results in Table (3) and Figure 5. Figures $8(\mathrm{~b})$ and $8(\mathrm{~d})$ project increasing HIV prevalence for increasing number of partnerships. Increased fluidity in mixing causes the general population to be exposed to higher risk from the core group thereby causing increased new infections. 


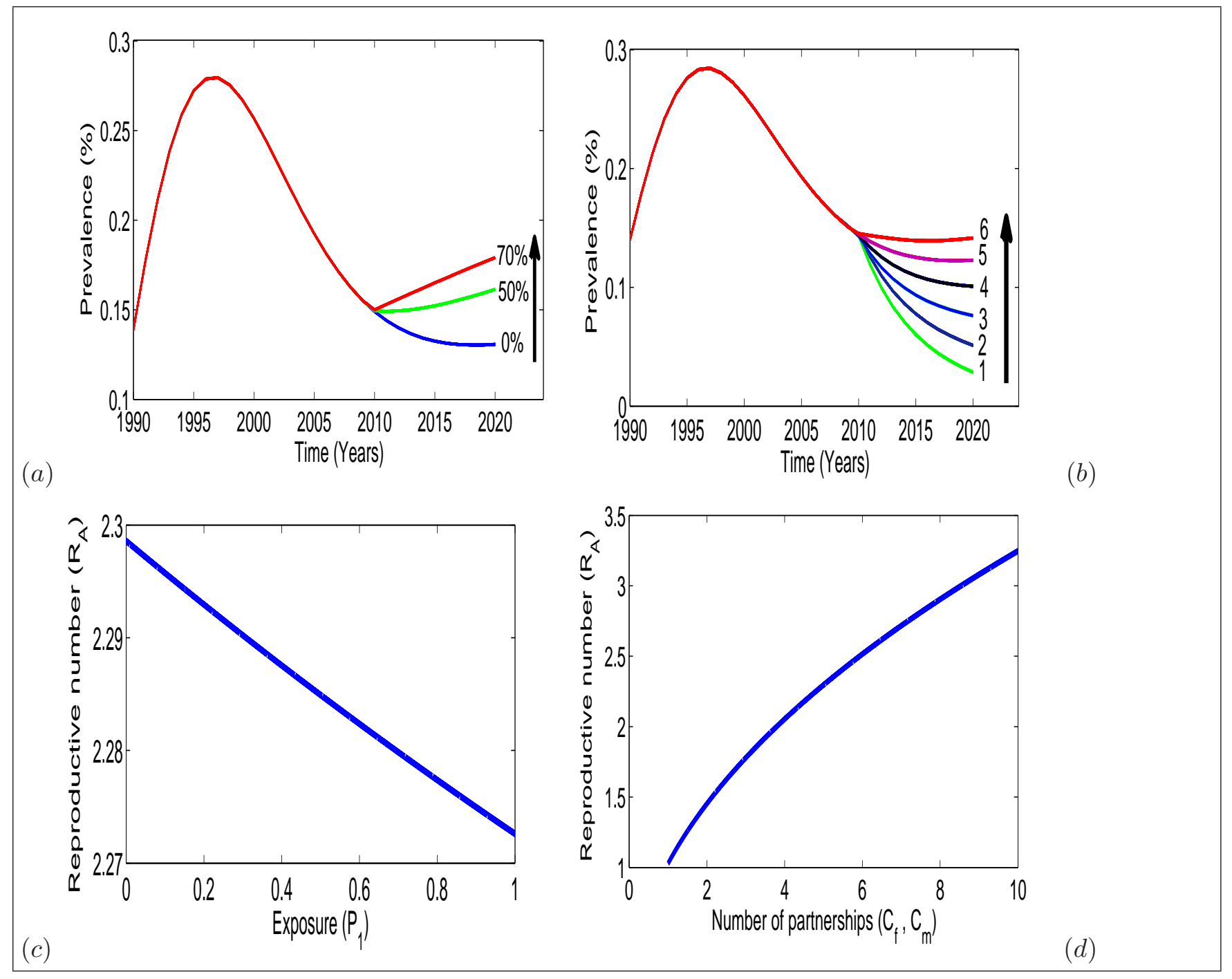

Figure 8: (a) Time series plot of HIV prevalence for increasing exposure to anal infection from baseline case $\left(1-p_{1}\right)=0$ to 0.7 . in the direction of the arrow, $(b)$ Time series plot of HIV prevalence for increasing number of partnerships $\left(c_{f}, c_{m}\right)=1$ to 6 in the direction of the arrow, $(c)$ Plot of the heterosexual AI induced reproductive number $\mathcal{R}_{A}$ against exposure to the risk of infection from vagina $p_{1},(d)$ Plot of the heterosexual AI induced reproductive number $\mathcal{R}_{A}$ against increasing number of partnerships $c_{f}$ and $c_{m}$.

\subsubsection{Estimating $\mathcal{R}_{A}$ at the start of the epidemic}

At the start of the epidemic, whether a disease becomes endemic or not, and its expected baseline or endemic level is usually not known. This may be true for emerging and/or recurring diseases which may have been absent from a community for a long time. The necessary control effort, quantified by the size of the reproductive number, is therefore usually unknown at the start. Using the next generation approach in [78], we derived the AI induced reproductive number

$$
\mathcal{R}_{A}=\sqrt{\mathcal{R}_{1}+\mathcal{R}_{2}+\mathcal{R}_{3}+\mathcal{R}_{4}+\mathcal{R}_{5}+\sqrt{\mathcal{R}_{1}^{2}+\mathcal{R}_{2}^{2}+\mathcal{R}_{3}^{2}+\mathcal{R}_{4}^{2}+\mathcal{R}_{5}^{2}+\mathcal{R}_{6}^{2}}}
$$

Derivation of $\mathcal{R}_{A}$ is shown in Appendix B. For the purpose of estimating $\mathcal{R}_{A}$ (equation (9)) at the start of the epidemic, we used a data point for 1989 from [55] and considered 1989 
to be the start of the epidemic and estimated $\mathcal{R}_{A}$ using observation data between 1989 and 1996. However we miss the important stage of the epidemic (1985-1989) in determining $\mathcal{R}_{A}$.

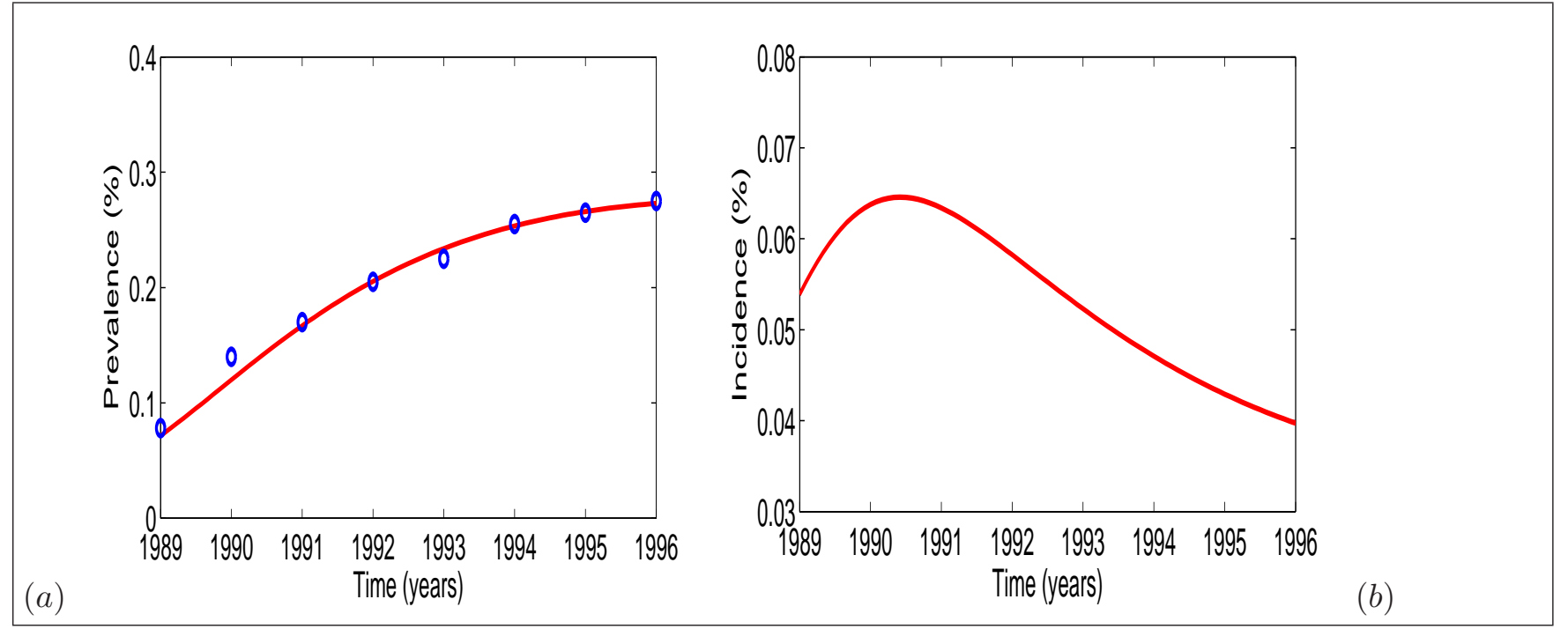

Figure 9: (a) Model fit to UNAIDS prevalence data point for Zimbabwe. The data for 1989 is taken from [55]. The basic reproductive number obtained from the fit $\mathcal{R}_{A}=4.28$. (b) Incidence curve corresponding to the prevalence from our model fit.

The model fit at the start of the epidemic gave us a value $\mathcal{R}_{A}=4.28$ which is slightly less than that obtained by [82] $\left(\mathcal{R}_{0}=5.10\right)$. Our estimate of $\mathcal{R}_{A}$ is severely curtailed by the fact that we used data from 1989 yet the first HIV case in Zimbabwe was detected in 1985. Whilst prevalence was increasing as shown in Figure 9(a) incidence had already started to decline from 1992 onwards as shown in Figure 9(b). The decline in incidence could be attributable to behaviour change due to experiential learning and therefore our value of $\mathcal{R}_{A}$ underestimates the magnitude of the control problem at the start of the epidemic.

\section{Discussion}

Several cross-sectional studies $[1,3,7,8,20,22,24,39,40,53]$ report the regular practice of heterosexual AI within various high-risk sub-populations in sub-Saharan Africa. In these subpopulations, heterosexual AI is episodic and routine component of sexual interaction [31]. We developed and analysed a heterosexual core group HIV model with risk of transmission through two competing modes of infection that is penile-vaginal and penile-anal pathways. The model was completely analytically resolved with disease elimination feasible whenever the reproductive number for the core group $\mathcal{R}_{c}$ falls below unity and disease persistence occuring whenever $\mathcal{R}_{c}$ exceeds unity. The core group model was then coupled to a general population model allowing core group individuals to interact freely with the general population without restriction. The combined model was fit to HIV data from UNAIDS [75] in order to parameterise the model and explore the parameter space related to heterosexual AI.

Using a Monte Carlo algorithm and Latin hypercube sampling-PRCC technique, we investigated the relative significance of individual parameters in influencing the size of the epidemic. It was shown that the core group size, the activity rates, the per partnership 
transmission probabilities for the general population as well as the proportion of infection risk associated with VI play a significant role in influencing the epidemic levels throughout our modelling span. These factors interplay to influence the epidemic size in a synergistic manner. Enhanced transmission rates (through AI), and high activity rates make the core group a reservoir for the virus, from which increasing contact rates ensure fluidity in mixing resulting in the bridging of the high risk core group and the low risk general population. These results from sensitivity analysis show that core group activity can potentially influence the HIV dynamics in the general population, a result also contained in [57].

The core group size was treated as a fit variable to assess the core group size most consistent with the data at hand. The data supported a core group size of between 5-20\%, a figure slightly elevated than results from observational studies $[29,53]$ estimating the core group size in Zimbabwe's neighboring countries at less than $1 \%$. Social stigmatisation is thought to possibly cause the under-reporting of $\mathrm{AI}$ in heterosexual populations $[44,66]$. Using the prevalence fit to data, an incidence curve was derived and both prevalence and incidence were projected through to calendar year 2020. Our incidence function shows incidence rising from a low base of $2.75 \%$ in 2005 to $3.25 \%$ in 2020 . It is usual in HIV modeling for the prevalence curve to lag behind the incidence curve and a rise in the later would certainly result in a rise in prevalence in 2020 unless current control effort is scaled up. Such phenomena may result from the strong dependency of infection rates on AIDS related deaths causing individuals to become complacent. Complacency occurs when individuals notice a dearth in AIDS related deaths and revert to risky behaviour such as condom non compliance. The phenomena poses one of the greatest challenges in the fight against HIV even in countries reporting plausible gains. Such phenomena has been observed in Uganda $[9,63]$ where national prevalence plummeted from $30 \%$ in the early 1980 s, to $18.3 \%$ in 1992 although some centers still recorded prevalence in excess of $30 \%$, falling from $15 \%$ in the early 1990s to $5 \%$ in 2001 and settling at $6.4 \%$ in 2006 and finally hitting $7.3 \%$ in 2011. Already Zimbabwe is reported to have recorded a $0,74 \%$ increase in HIV prevalence from $14.26 \%$ recorded in 2011 to $15 \%$ in 2012 [19].

Studies report prevalence of heterosexual AI outside of Africa at around 20\% [53]. This is happening in the context of low to zero HIV prevalence. To assess the potential impact of heterosexual AI on HIV dynamics in higher HIV prevalence settings, we thus increased the core group size to $20 \%$ in 2010 . Prevalence and incidence were then projected to the year 2020 by varying exposure to AI from $0 \%$ to $70 \%$. Equal exposure to the risk of both anal and vaginal infection would result in HIV prevalence rising up by $23 \%$ from the baseline values in year 2020. An increase in the core group size and hence the incidence of heterosexual AI thus has the potential to amplify the HIV epidemic. The possibility of increasing numbers of people engaging in heterosexual AI in the future, reversing the gains recorded thus far against the HIV scourge is real. HIV policy objectives must therefore include informing heterosexuals on the dangers of engaging in AI which presents higher infection risks.

In a heterosexual community at risk of infection from AI, the risk of exposure to infection from AI is potentially, a modifiable factor in the reduction of HIV infections. Results showed that in communities where heterosexual AI is prevalent, reduced exposure to infection via AI would lower disease prevalence. Risk of infection is significantly increased during AI and minimising the frequency and duration of heterosexual AI as well as withdrawal before ejaculation (which is known to increase transmission to the receptive partner) if infective partner is insertive, will assuage the risk of infection from penile-AI. Exposure risk 
reduction is achievable through educational and awareness programmes specifically targeted at heterosexuals and these may include strategic positioning at the time of ejaculation if the insertive partner is seropositive. Other risk mitigating strategies to consider are those currently used by MSM that is; negotiated safety (a pact between HIV individuals to restrict unprotected sex within a regular partnership), withdrawal and serosorting (limiting AI only to partners with concordant HIV status) [33,34]. Some implements to reduce infectivity during heterosexual AI such as lubricants $[17,41,70]$, microbicides $[12,51,52,70]$ and condoms $[32,69]$ can be considered and these have already proved useful as preventive measures amongst MSM.

For emerging and/or recurring diseases which may have been absent from a community for a long time, it is not easy to predict baseline levels at the start of the epidemic. Using the model fit to data, we estimated the reproductive number at the beginning of the epidemic in Zimbabwe at $\mathcal{R}_{A}=4.40$ which is lower than that of [82] $(\mathcal{R}=5.10)$. By year 1992, HIV incidence was already on the decline and there was evidence of behaviour change due to AIDS deaths experiences. Thus our estimate of $\mathcal{R}_{A}$ at the start of the epidemic is handicapped by the lack of data from 1985 (the year of first case detection) to 1990 when sentinel data first became available.

We quantified and segregated the incidence due to the specific infection pathways. Although the study results support the penile-vaginal pathway as the main source of infection, apportioning the incidence to its source puts emphasis on the necessity for public health practitioners to target specific infection pathways for intervention. The risk of AI is acknowledged in MSM but is ignored in heterosexual populations. The implements to mitigate risk during AI are already available and in use amongst MSM as well as risk reduction strategies. What is left is acceptance in policy making that low heterosexual AI activity in the region is occurring in the context of high HIV prevalence settings and increasing AI prevalence has the potential of impacting upon HIV dynamics in heterosexual populations with generalised epidemics. Public health objectives should include crafting a policy that promotes risk reduction such as consistent condom use, reduction of the frequency of unprotected AI and of gender inequities which have been cited in literature as increasing the incidence of heterosexual AI. The need for sociological studies around the subject of heterosexual AI with a view to perforating the secrecy veil which envelopes the social dynamic is made more compelling by modelling results from this study.

Acknowledgments:Malunguza acknowledges the support of the National University of Science and Technology. The authors acknowledge Dr Christinah Mukandavire for useful comments and discussions on the manuscript, Dr Sarudzai Portia Showa for helpful input on numerics and the anonymous reviewers whose input have improved this work tremendously.

\section{References}

[1] Abdool K. Q., Karim S. S., Soldan K., Zondi M., Reducing the risk of HIV infection among South African sex workers: socioeconomic and gender barriers, American Journal of Public Health, 85, 1521-1525, (1995).

[2] AIDS.gov, Stages of HIV infection, (2013), www.aids.gov [Accessed 23 June 2016]. 
[3] Akande A., AIDS-related beliefs and behaviors of students: evidence from two countries (Zimbabwe and Nigeria), International Journal of Adolescent Youth, 4, 285-303, (1994).

[4] Auvert B., Marais D., Lissouba P., Zarca K., Ramjee G., Williamson A. L., High-risk human papillomavirus is associated with HIV acquisition among South African female sex workers, Infectious Diseases in Obstetrics and Gynecology, (2011).

[5] Baldwin J. I. and Baldwin J. D., Heterosexual Anal Intercourse: An Understudied, High-Risk Sexual Behavior, Archives of Sexual Behavior, (29)4, 357-373 (2000).

[6] Baggaley F. R., White R. G., Boily M., HIV transmission risk through anal intercourse: systematic review, meta-analysis and implications for HIV prevention, International Journal of Epidemiology, 39, 1048-1063, (2010).

[7] Baggaley R. F., White R. G., and Boily M. C., Systematic review of orogenital HIV-1 transmission probabilities, International Journal of Epidemiology, 37(6), 1255-1265, (2008).

[8] Baggaley R. F., Dimitrov D., Owen B. N., Pickles M., Butler A. R, Masse B., Boily MC. Heterosexual anal intercourse: a neglected risk factor for HIV?, American Journal of Reproductive Immunology, 69 (1), 95-105, (2013).

[9] Baryarama F., Luboobi, L. S. and Mugisha J. Y. T. 2006, Mathematical Model for HIV/AIDS with Complacency in a population with declining prevalence. Computational and Mathematical Methods in Medicine, 7(1), 27-35, (2006).

[10] Bingenheimer J. B., Men's multiple sexual partnerships in 15 Sub-Saharan African countries: Sociode-mographic patterns and implications, Studies in Family Planning, 41, 1-17, (2010).

[11] Boily M. C., The relative contribution of anal intercourse and primary infection to mature heterosexual epidemics, Sexually Transmitted Infections, 87(1), A38, (2011).

[12] Boily M. C., Dimitrov D., Abdool Karim S. S., Masse S., The future role of rectal and vaginal microbicides to prevent HIV infection in heterosexual populations: implications for product development and prevention, Sexually Transmitted Infections, 87, 646-653, (2011).

[13] Bongaarts J., A model for the spread of HIV infection and the demographic impact of AIDS, Statistical Medicine, 8, 103-120, (1989).

[14] Brauer, F., Castillo-Chavez, C. Mathematical models in population biology and epidemiology. In Texts in Applied Mathematics Series Springer-Verlag, New York. (2001).

[15] Busenberg S. and van den Driessche P., Analysis of a disease transmission model in a population with varying size, Journal of Mathematical Biology, 28, 257-270, (1990).

[16] Centre for Communicable Diseases and Infection Control, HIV Transmission Risk: A Summary of Evidence, http://www.phac-aspc.gc.ca/aids-sida/publication/hivtr-rtviheng.php, accessed 25-07-2013.

[17] Chateau M. L., Denton P. W., Swanson M. D. et al., Rectal transmission of transmitted/founder HIV-1 is efficiently prevented by topical $1 \%$ tenofovir in BLT humanized mice. PloS One, 8(3), e60024, (2013). 
[18] Cohen M. S., Miller W. C., Sexually transmitted diseases and human immunodeficiency virus infection: cause, effect, or both?, International Journal of Infectious Diseases, 3(1), 14, (1998).

[19] Chronicle, Zim HIV prevalence rate rises to 15pc, http://www.chronicle.co.zw/zimhiv-prevalence-rate-rises-to-15pc/.Accessed 15-11-2013.

[20] Decker M. R., Seage G. R., Hemenway D., Gupta J., Raj A., Silverman J. G., Intimate partner violence perpetration, standard and gendered STI/HIV risk behavior, and STI/HIV diagnosis among a clinic-based sample of men, Sexually Transmitted Infections, 85, 555-560, (2009).

[21] Duby Z., Hartmann M., Montgomery E. T., Colvin C. J., Mensch B., van der Straten A., Sexual scripting of heterosexual penile-anal intercourse amongst participants in an HIV prevention trial in South Africa, Uganda and Zimbabwe, Culture Health and Sexuality, 18(1), 30-44, (2016).

[22] Erickson P. I., Bastani R., Maxwell A.E., Marcus A.C., Capell F.J. and Yan K. X., Prevalence of anal sex among heterosexuals in Califorinia and its relationship to other AIDS risk behaviors, AIDS, Education and Behaviour, 7(6), 477-493, (1995).

[23] European Study Group on Heterosexual Transmission of HIV, Comparison of female to male and male to female transmission of HIV in 563 stable couples, BMJ, 304(6830), 809-813, (1992).

[24] Flisher A. J., Ziervogel C. F., Chalton D. O., Leger P. H., Robertson B. A., Risktaking behavior of Cape Peninsula high-school students, Part VIII, Sexual behavior. South African Medical Journal, 83, 495-497, (1993).

[25] Guidelines for the management and post exposure prophylaxis of individuals who sustain nonoccupational exposure to HIV, ANCAHRD/CTARC Bulletin, (2001).

[26] Hsieh Y. and Chen C. H., Modelling the Social Dynamics of a Sex Industry: Its Implications for Spread of HIV/AIDS, Bulletin of Mathematical Biology, 66, 143-166, (2004) .

[27] Gisselquist D., Potterat J. J., Heterosexual transmission of HIV in Africa: an empiric estimate, International Journal of STD AIDS., 14(3), 162-73, (2003).

[28] Granich R. M., Gilks C. F., Dye C., De Cock K. M., Williams B. G., Universal voluntary HIV testing with immediate antiretroviral therapy as a strategy for elimination of HIV transmission: a mathematical model, 373, 48-57, Lancet, (2009).

[29] Gray R. H, Wawer M. J., Brookmeyer R., Probability of HIV-1 transmission per coital act in monogamous, heterosexual, HIV-1-discordant couples in Rakai, Uganda, The Lancet, 357(9263), 1149-1153, (2001).

[30] Gregson S., Garnett G. P., Shakespeare R., Foster G. and Anderson R. M., Determinants of the demographic impact of HIV-1 in sub-Saharan Africa: the effect of a shorter mean adult incubation period on trends in orphanhood, Health Transition Review, AIDS Impact and Prevention in the Developing World: Demographic and Social Science Perspectives, (4), 65-92, (1994). 
[31] Griensven F. de Vroome E. M. M., Tielman R. A. P et al., Impact of HIV antibody testing on changes in sexual behavior among homosexual men in The Netherlands, American Journal of Public Health, 78, 1575-1577, (1998).

[32] Griensven F, Non-condom use risk-reduction behaviours: can they help to contain the spread of HIV infection among men who have sex with men? AIDS, 23, 253-255, (2009).

[33] Healthy Communities, HIV prevention risk reduction strategies, www.healthycommunities.org.au, accessed 24-06-2015.

[34] Holt M. and Ellard J., HIV risk reduction strategies among gay men for the Australian Federation of AIDS Organisations. ISBN: 978187646948 X., (2011), http://www.afao.org.au (accessed 24 June 2015).

[35] Hyman J. M., Li J., Stanley E. A., The differential infectivity and staged progression models for the transmission of HIV, Mathematical Biology, 155, 77-109, (1999).

[36] Hyman J. M., Li J., Stanley E. A., The initialization and sesitivity of multigroup models for the transmission of HIV, Journal of Theoretical Biology, 208, 2217-249, (2001).

[37] Jina F., Janssona J., Lawa M., Prestage G. P., Zablotska I et al. al. Per-contact probability of HIV transmission in homosexual men in Sydney in the era of HAART, AIDS, 24, 907-913, (2010).

[38] Johnson E. H., Gilbert D., and Lollis C., Characteristics of African-American college students with HIV/AIDS. Journal of the National Medical Association, (86) 931-940, (1994).

[39] Kalichman S. C., Pinkerton S. D., Michael P. C., Demetria C., Mehlomakulu V., Carey K., Simbayi L. C., Mwaba K. and Harel O., Heterosexual anal intercourse and HIV infection risks in the context of alcohol serving venues, Cape Town, South Africa, BMC Public Health, 11, 807, 2011, http://www.biomedcentral.com/, accessed 24-07-2013.

[40] Kalichman S. C., Simbayi L., Cain D., Jooste S., Heterosexual anal intercourse among community and clinical settings, Cape Town, South Africa, Sexually Transmitted Infections, 85(6), 411-5, (2009).

[41] Karim Q. A., Karim S. S. A., Frohlich J. A., et al., Effectiveness and safety of tenofovir gel, an antiretroviral microbicide, for the prevention of HIV infection in women, Science, 329(5996), 1168-1174, (2010).

[42] Kassem T. G., Roudenko S., Tennenbaum S., Castillo-Chavez C., The Role of Transactional Sex in the Spread of HIV /AIDS: A Modeling Perspective, Mathematical and Theoretical Biology Institute, (2005).

[43] Kaestle C. E., Halpern, C. T., Whats love got to do with it? Sexual behaviors through emerging adulthood, Perspectives on Sexual and Reproductive Health, (39), 134-140, (2007).

[44] Kloos H., Mariam D. H., Some neglected and emerging factors in HIV transmission in Ethiopia, Ethiop Med J., (45), 103-107, (2007). 
[45] Kristen L. et al., Intercourse In Young Adult Heterosexual Relationships, Perspectives on Sexual and Reproductive Health, 45(1), 612, (2013).

[46] Lee M., Roman C., Wiley S., Hernandez Suarez C., Kribs Zaleta C., Oliva R., The effect of the HIV / AIDS epidemic on the population of truck drivers in South Africa and its economic impact, Cornell University, (2002).

[47] Leichliter J. S., Chandra A., Liddon N., Fenton K. A., Aral S. O., Prevalence and correlates of heterosexual anal and oral sex in adolescents and adults in the United States, The Journal of Infectious Diseases, 196(12), 1852-9, (2008).

[48] Leynaert B., Downs A. M., de Vincenzi I., Heterosexual transmission of human immunodeficiency virus: variability of infectivity throughout the course of infection, European Study Group on Heterosexual Transmission of HIV, American Journal of Epidemiology, 148(1), 88-96, (1998).

[49] MacDonald N. E., Wells G. A., Fisher W. A., Warren W. K., King M. A., Doherty J. A. and Bowie W.R., High-risk STD/HIV Behavior Among College Students, Journal of the American Medical Association, 263, 3155-3159, (1990).

[50] McBride K. R. and Fortenberry J. D., Heterosexual Anal Sexuality and Anal Sex Behaviors: A Review, Journal of Sex Research, 47(23), 123-136, (2010).

[51] McGowan I., Rectal microbicides: Can we make them and will people use them? Aids and Behavior, 15(1), S66-S71, (2011).

[52] McGowan I., McGowan I. Rectal microbicide development, Current Opinion HIV $A I D S, 7(6)$, 526-533, (2012).

[53] Makhubele B. and Parker W., Heterosexual anal sex amongst young adults in South Africa: Risks and perspectives, www.cadre.org.za, accessed 10-05-2013.

[54] Mannava P., Geibel S., Kingola N., Temmerman M., Luchters S., Male sex workers who sell sex to men also engage in anal intercourse with women: evidence from Mombasa, Kenya, PLoS ONE, 8(1), (2013).

[55] Ministry of Health and Child Welfare, National AIDS Coordination Programme, HIV/AIDS in Zimbabwe:Background, Projections, Impact and Interventions National AIDS Coordination Programme, 1998, www.policyproject.com/pubs/countryreports/Zimaim.pdf?, accessed 17-11-2013.

[56] Ministry of Health and Child Welfare, Zimbabwe National HIV and AIDS Estimates, (2003), http://www.sarpn.org/documents/d0000989/P1107-PNADA504.pdf, accessed 27-08-2013.

[57] Moghadas S. M., Two core group models for sexual transmission of disease, Ecology Modelling, 148, 15-26, (2002).

[58] Mukandavire Z., A theoretical assessment of HIV/AIDS intervention strategies with some application to Zimbabwe. PhD thesis, National University of Science and Technology, Bulawayo, Zimbabwe, (2006).

[59] Mukandavire Z. and Garira W., HIV/AIDS model for assessing the effects of prophylactic sterilizing vaccines, condoms and treatment with amelioration, Journal of Biological Systems, 14(3), 323-355, (2006). 
[60] Naresh R., Tripathi A. and Sharma D., Modelling and analysis of the spread of AIDS epidemic with immigration of HIV infectives. Mathematical and Computational Modelling, 49, 880-892, (2009a).

[61] Naresh R., Tripathi A. and Sharma D., Modelling the effect of risky sexual behaviour on the spread of HIV/AIDS, International Journal of Applied Mathematics and Computation, 1(3), 132-147, (2009b).

[62] Ndinda C., Chimbwete C., Mcgrath N. and Pool R., Perceptions of anal sex in rural South Africa, Culture, Health and Sexuality, (10)2, 205-212, (2008).

[63] Ogwang J., Complacency, low funding take a toll as HIV prevalence hits 7.3\%, (2013), http://www.newvision.co.ug/news/643728-complacency-low-funding-take-a-toll-ashiv-prevalence-hits-7-3.html, accessed 20-10-2013.

[64] Osmond D. H., Epidemiology of Disease Progression in HIV, (1998), http://hivinsite.ucsf.edu, accessed 23-06-2015.

[65] Padian N. S., Shiboski S. C., Glass S. O., Vittinghoff E., Heterosexual transmission of human immunodeficiency virus (HIV) in Northern California: results from a ten-year study, American Journal of Epidemiology, 146, 350-357, ( 1997).

[66] Smith L. B., Adler N. E., Tschann J. M., Underreporting sensitive behaviors: the case of young women's willingness to report abortion, Health Psychology, (18), 37-43, (1999).

[67] Reynolds G. L., Fisher D. G. and Rogala B., Why Women Engage in Anal Intercourse: Results from a Qualitative Study, Archives of Sexual Behavior, 44(4), 983-995, (2015).

[68] Royce R. A., Sena A., Cates W., Cohen M.,S., Sexual transmission of HIV, New England Journal of Medicine 336(15),1072-1078 (1997).

[69] Smith D. K., Herbst J. H., Zhang X., Rose C. E., Condom efficacy by consistency of use among men who have sex with men (MSM) in the United States. 20th Conference on Retroviruses and Opportunistic Infections, Atlanta, GA, USA. March 6, (2013), http://www.aidsmap.com, accessed 24-06-2015.

[70] Stone E., Heagerty P., Vittinghof E. et al., Correlates of condom failure in a sexually active cohort of men who have sex with men, Journal of Acquired Immune Deficiency Syndrome Human Retrovirology, 9, 495-501, (1999).

[71] Tanser F., Bärnighausen T., Hund L., Garnett G. P., McGrath N. and Newell M., Effect of concurrent sexual partnerships on rate of new HIV infections in a high-prevalence, rural South African population: a cohort study The Lancet, 378(9787), 247-255, (2011).

[72] Tennenbaum S., Kassem T. G., Roudenko S., Castillo-Chavez C., The role of transactional sex in spreading HIV in Nigeria, in Mathematical studies on human disease dynamics: emerging paradigms and challenges : AMS-IMS-SIAM Joint Summer Research Conference on modeling the dynamics of human diseases : emerging paradigms and challenges, American Mathematical Society, (410), 2006.

[73] UNAIDS, Evidence for HIV decline in Zimbabwe: A comprehensive review of the epidemiological data, (2005), http://data.unaids.org. 
[74] UNAIDS: Global report: UNAIDS report on the global AIDS epidemic. 2010, UNAIDS/10.11E JC1958E, http://www.unaids.org, accessed 24-07-13.

[75] UNAIDS fact sheets, (www.unaids.org), (accessed 31-08-2012).

[76] UNAIDS, Zimbabwe national HIV and AIDS strategic plan (ZNASP), 2006-2010, http://www.safaids.net/files/ZNASP\%202006-2010.pdf, accessed 27-08-2013.

[77] UNICEF, Migration profiles, https://esa.un.org/miggmgprofiles/indicators/files/Zimbabwe.pdf.

[78] van den Driessche P. and Watmough J., Reproduction numbers and sub-threshold endemic equilibria for compartmental models of disease transmission, John A. Jacquez memorial volume, Mathematical Biosciences, (180), 29-48, (2002).

[79] Varghese B, Maher J. E., Peterman T. A., Branson B. M., Steketee R. W., Reducing the risk of sexual HIV transmission: quantifying the per-act risk for HIV on the basis of choice of partner, sex act, and condom use, Sexually Transmitted Diseases, 29(1), 38-43, (2002).

[80] Voeller B., AIDS and heterosexual anal intercourse, Archives of Sexual Behaviour, 20, 233-276 (1991).

[81] Wawer M. J., Gray R. H., Sewankambo N. K., Serwadda D., Li X., Laeyendecker O., Kiwanuka N., Kigozi G., Kiddugavu M., Lutalo T., Nalugoda F., Wabwire-Mangen F., Meehan M. P., Quinn T. C., Rates of HIV-1 transmission per coital act by stage of HIV-1 infection in Rakai, Uganda, The Journal of Infectious Diseases, 191, 1403-9, (2005).

[82] Williams B. G., Gouws E., $\mathcal{R}_{0}$ and the elimination of HIV in Africa, Cornell University Libray, (2013).

[83] Williams B. G., Lloyd-Smith J. O., Gouws E. et al., The potential impact of male circumcision on HIV in Sub-Saharan Africa, PLoS Medicine, 3:e262, (2006).

[84] Williams B. G., Korenromp E. L., Gouws E., Schmid G. P., Auvert B., Dye C., HIV infection, antiretroviral therapy, and CD4+ cell count distributions in African populations, Journal Infectious Diseases, 194, 1450-58, (2006).

[85] Zhien M. and Liu J., Stability analysis for differential infectivity epidemic models, Nonlinear Analysis, 4, 841-856, (2003).

\section{Appendix A}

A study by [7] estimated anal transmission probabilities using a similar Bernoulli process that assumes independence of risk of transmission per sex act within a partnership:

$$
\beta_{p, a}=1-\left(1-\beta_{c, a}\right)^{n},
$$

where $\beta_{p, a}$ and $\beta_{c, a}$ are the per partner $(p)$ and per-act $(c)$ transmission probability for AI. For heterosexual populations practicing both vaginal and AI they came up with a single transmission probability per partnership and equation (A.1) transforms to

$$
\beta_{p, a l l}=1-\left(1-\beta_{c, v}\right)^{(1-d) n}\left(1-\beta_{c, a}\right)^{d n},
$$


where $\beta_{p, \text { all }}$ is risk per-partner for VI and AI,

$\beta_{c, v}$ and $\beta_{c, a}$ are per act transmission probabilities for VI and receptive AI respectively and $d$ is the proportion of $n$ sex acts which are anal acts rather than vaginal acts. Thus this formulation assumes that VI and AI acts are mutually exclusive events which do not occur simultaneously in our definition of a single sex act. Such assumption may at least double the contact number given that heterosexual couples may intermittently shift between VI and AI during a single sex act and thus in our case is not suitable for relating contacts to number of partners per sex act.

\section{Appendix B}

Model system (8) has a disease-free equilibrium given by, $\xi_{0}$ where

$$
\left.\xi_{0}=\left(\frac{\Lambda \pi_{0}}{(\alpha+\mu+\psi)}, 0, \frac{\Lambda\left(1-\pi_{0}\right)}{(\alpha+\mu+\psi)}, 0, \frac{\Lambda \psi \pi_{0}}{(\alpha+\mu)(\alpha+\mu+\psi)}, 0, \frac{\Lambda \psi\left(1-\pi_{0}\right)}{(\alpha+\mu)(\alpha+\mu+\psi)}\right), 0,0\right) .
$$

Following van den Driessche and Watmough [78], the heterosexual AI induced reproductive number for system (8), $\mathcal{R}_{A}$ is given by the spectral radius of the matrix $F V^{-1}$ where the $F$ and $V$ matrices are given by,

$$
F=\left(\begin{array}{cccc}
0 & 0 & \frac{(\alpha+\mu) c_{f} \pi_{0} \beta_{m_{v}}}{\theta\left(1-\pi_{0}\right)} & \frac{(\alpha+\mu) c_{f} \pi_{0} \beta_{m_{v}}}{\theta\left(1-\pi_{0}\right)} \\
\frac{(\alpha+\mu) c_{m}\left(1-\pi_{0}\right) \beta_{f_{v}}}{(\alpha+\mu+\psi) \pi_{0}} & \frac{(\alpha+\mu) c_{m}\left(1-\pi_{0}\right) \beta_{f_{v}}}{\theta \pi_{0}} & 0 & 0 \\
0 & 0 & \frac{\psi c_{f} \pi_{0} \beta_{m_{v}}}{\theta\left(1-\pi_{0}\right)} & \frac{\psi c_{f} \pi_{0} \beta_{m}^{\prime}}{\theta\left(1-\pi_{0}\right)} \\
\frac{\psi c_{m}\left(1-\pi_{0}\right) \beta_{f_{v}}}{\theta \pi_{0}} & \frac{\psi c_{m}\left(1-\pi_{0}\right) \beta_{f}^{\prime}}{\theta \pi_{0}} & &
\end{array}\right),
$$

and

$$
V=\left(\begin{array}{cccc}
(\alpha+\gamma+\mu+\psi) & 0 & 0 & 0 \\
0 & 0 & (\alpha+\gamma+\mu+\psi) & 0 \\
\psi & (\alpha+\gamma+\mu) & 0 & 0 \\
0 & 0 & \psi & (\alpha+\gamma+\mu)
\end{array}\right)
$$

where $\left.\theta=(\alpha+\gamma+\psi), \beta_{m}^{\prime}=\left(1-p_{1}\right) \beta_{m_{a}}-p_{1} \beta_{m_{v}}\right)$ and $\left.\beta_{f}^{\prime}=\left(1-p_{1}\right) \beta_{f_{a}}-p_{1} \beta_{f_{v}}\right)$. Thus $\mathcal{R}_{A}=$ $\sqrt{\mathcal{R}_{1}+\mathcal{R}_{2}+\mathcal{R}_{3}+\mathcal{R}_{4}+\mathcal{R}_{5}+\sqrt{\mathcal{R}_{1}^{2}+\mathcal{R}_{2}^{2}+\mathcal{R}_{3}^{2}+\mathcal{R}_{4}^{2}+\mathcal{R}_{5}^{2}+\mathcal{R}_{6}^{2}}}$ where the partial reproductive num- 
bers that track the movement of an infective over their infectivite lifetime are given by,

$$
\left\{\begin{array}{l}
\mathcal{R}_{1}=\frac{c_{f} c_{m} \beta_{f_{a}} \beta_{m_{a}} \psi^{2}\left(1-p_{1}\right)^{2}}{2(\alpha+\gamma+\mu)^{2}(\alpha+\mu+\psi)^{2}}, \\
\mathcal{R}_{2}=\frac{c_{f} c_{m} \beta_{f_{a}} \beta_{m_{v}} \psi^{2}\left(1-p_{1}\right)\left(\alpha+\mu+(\alpha+\gamma+\mu+\psi) p_{1}\right)}{2(\alpha+\mu+\gamma)^{2}(\alpha+\mu+\psi)^{2}(\alpha+\mu+\gamma+\psi)^{2}}, \\
\mathcal{R}_{3}=\frac{c_{f} c_{m} \beta_{f_{a}} \beta_{m_{a}} \psi^{2}\left(1-p_{1}\right)\left(\alpha+\mu+(\alpha+\gamma+\mu+\psi) p_{1}\right)}{2(\alpha+\mu+\gamma)^{2}(\alpha+\mu+\psi)^{2}(\alpha+\mu+\gamma+\psi)^{2}}, \\
\mathcal{R}_{4}=\frac{\left.c_{f} c_{m} \beta_{f_{v}} \beta_{m_{v}}(\alpha+\mu)(\mu+2 \psi)+\mu(\mu+3 \psi)+\alpha(\gamma+2 \mu+3 \psi)\right)}{2(\alpha+\mu+\gamma)^{2}(\alpha+\mu+\psi)^{2}(\alpha+\mu+\gamma+\psi)^{2}}, \\
\mathcal{R}_{5}=\frac{c_{f} c_{m} \beta_{f_{v}} \beta_{m_{v}} \psi^{2} p_{1}\left(2(\alpha+\mu)+(\alpha+\gamma+\mu+\psi) p_{1}\right)}{2(\alpha+\mu+\gamma)^{2}(\alpha+\mu+\psi)^{2}(\alpha+\mu+\gamma+\psi)^{2}}, \\
\mathcal{R}_{6}=\frac{c_{f} c_{m} \beta_{f_{v}} \beta_{m_{v}}(\alpha+\mu)^{2}\left(1-p_{1}\right)^{2}\left(\beta_{f_{a}}-\beta_{f_{v}}\right)\left(\beta_{m_{a}}-\beta_{m_{v}}\right)}{2(\alpha+\mu+\gamma)^{2}(\alpha+\mu+\psi)^{2}(\alpha+\mu+\gamma+\psi)^{2}} .
\end{array}\right.
$$

